TRANSACTIONS OF THE

AMERICAN MATHEMATICAL SOCIETY

Volume 339, Number 2, October 1993

\title{
NONCOMMUTATIVE KÖTHE DUALITY
}

\author{
PETER G. DODDS, THERESA K.-Y. DODDS, AND BEN DE PAGTER
}

Dedicated to W. A. J. Luxemburg on the occasion of his 63rd birthday

\begin{abstract}
Using techniques drawn from the classical theory of rearrangement invariant Banach function spaces we develop a duality theory in the sense of Köthe for symmetric Banach spaces of measurable operators affiliated with a semifinite von Neumann algebra equipped with a distinguished trace. A principal result of the paper is the identification of the Köthe dual of a given Banach space of measurable operators in terms of normality.
\end{abstract}

\section{INTRODUCTION}

The study of rearrangement invariant spaces of measurable functions has long been of central importance in many branches of real analysis as is clear from the monograph of Krein, Petunin, and Semenov [KPS] and the paper of Luxemburg [Lu]. Of equal importance in the study of spaces of compact operators in Hilbert space is the notion of singular value [GK] which, for a given compact operator plays a similar role to that of the decreasing rearrangement of a measurable function. These ideas admit a common extension in the notion of a generalized decreasing rearrangement of an arbitrary selfadjoint operator affiliated with a given semifinite von Neumann algebra (with a distinguished trace) which has proved fruitful in many contexts [Gr, Ov1, 2, 3, FA1, 2, FK, Ye2, 3], among others. Based on the theory of noncommutative integration introduced by Segal [Se], Ovčinnikov [Ov1, 2] showed that the setting of semifinite von Neumann algebras with a trace provides a natural setting for various interpolation theorems, unifying in particular work of Calderón [Ca] in spaces of measurable functions with analogous results of Russu [Ru] for trace ideals. Of central importance in this work is the role played by the rearrangement invariant structure. Similar ideas occur in the subsequent work of Yeadon [Ye2, 3] motivated by the classical theory of Banach function spaces in the sense of Luxemburg and Zaanen [Lu, Za1]. More recently, a method of construction of rearrangement invariant Banach spaces of measurable operators has been given in [DDP1, 2] which is considerably more general than that permitted by the methods of [Ov2, Ye3] and it is the intention of this paper to develop these

Received by the editors January 23, 1991 and, in revised form, July 9, 1991.

1991 Mathematics Subject Classification. Primary 46E30; Secondary 46L50, 47B55.

Key words and phrases. Rearrangement invariant Banach function spaces, measurable operators, Köthe duality, normality.

The second author's research was supported by A.R.C. 
ideas further, particularly from the point of view of Köthe duality. For ideals of compact operators, this study has been initiated by Garling [Ga] and, from the present viewpoint, by Yeadon [Ye2, 3]. As will become apparent, the techniques needed to establish a satisfactory theory of Köthe duality for spaces of measurable operators depend crucially on certain methods of interpolation theory related to rearrangement inequalities and to the classical theory of doubly stochastic matrices, and the earlier results of $[\mathrm{Ov} 2, \mathrm{Ye} 2,3]$ in these directions do not suffice for our present purposes. In particular, it is necessary to point out that several key arguments in [Ye2, 3] are not valid as stated. For basic properties of decreasing rearrangements of measurable operators (for precise definitions see $\S 1$ below), we refer to the paper of Fack and Kosaki [FK], but otherwise the discussion of the present paper is self-contained for the sake of unity of method. The first three sections are concerned with the order structure of the space of all measurable operators affiliated with a given semifinite von Neumann algebra and various properties of normed rearrangement invariant operator spaces. These preliminary results complement the discussion of [FK] and indicate how the present approach via decreasing rearrangements coincides in familiar examples with other approaches already in the literature as, for example, that given by Nelson $[\mathrm{Ne}]$ or those given in the monographs [Di, Ta, SZ], to which we refer as convenient references concerning von Neumann algebras. As in [FK], we adopt throughout the notion of measurability introduced by Nelson [Ne] which is perhaps more naturally adapted to the present context than the notion of measurability due to Segal [Se] which requires more detailed structural knowledge of von Neumann algebras. In fact, for closed operators affiliated with a semifinite von Neumann algebra equipped with a normal faithful semifinite trace $\tau$, the notion of $(\tau$-)measurability in the sense of Nelson is equivalent to requiring the existence of an everywhere finite decreasing rearrangement.

The machinery essential to the subsequent duality theory is given in $\S 4$ and here the principal result (Theorem 4.7) is an exact noncommutative extension of a characterization due to Calderón of the image of a measurable function under operators which are simultaneous contractions for the $L^{1}$ and $L^{\infty}$-norms in terms of a preorder relation which goes back to Hardy, Littlewood, and Pólya in the context of doubly stochastic matrices. The present approach is based on a refinement of a rearrangement inequality due to Hardy and Littlewood (Theorem 4.5) and follows the ideas suggested by Fremlin [Fr] in the commutative setting. The basic Köthe duality theory is presented in $\S 5$ and one of the main results (Theorem 5.11) is a characterization of the Köthe dual of a given noncommutative space via a Radon-Nikodym type theorem and is an extension of the well-known fact that the predual of a von Neumann algebra may be identified with that subspace of the Banach dual consisting of normal linear functionals. Our method is a considerable refinement of an earlier result of Yeadon [Ye2, 3] and implies not only the usual duality results for the more familiar $L^{p}$-spaces but also applies readily to the identification of the Banach dual spaces of more general spaces of measurable operators. The paper concludes with an exact extension of the well-known theorem of G. G. Lorentz and W. A. J. Luxemburg concerning the isometric embedding of a Banach function space into its Köthe bidual which yields in turn an extension of a reflexivity criterion of $\mathrm{T}$. Ogasawara. 
Some of the results of this paper were presented in May 1989 at the Tagung Riesz Spaces and Operators, Mathematisches Forschungsinstitut, Oberwolfach.

The authors wish to thank the referee for several useful comments.

\section{ORDER PROPERTIES OF MEASURABLE OPERATORS}

Throughout this paper, we denote by $\mathscr{M}$ a semifinite von Neumann algebra in the Hilbert space $H$ with given normal faithful semifinite trace $\tau$. The identity in $\mathscr{M}$ is denoted by 1 , and we denote by $\mathscr{M}^{p}$ the set of all (selfadjoint) projections in $\mathscr{M}$. The closed, densely defined linear operator $x$ in $H$ with domain $D(x)$ is said to be affiliated with $\mathscr{M}$ if and only if $u^{*} x u=x$ for all unitary operators $u$ belonging to the commutant $\mathscr{M}^{\prime}$ of $\mathscr{M}$. The preceding operator equality is to be understood in the sense that $u^{*} x u, x$ have the same domain so that $u(D(x))=D(x)$. If the linear operator $x$ is affiliated with $\mathscr{M}$, then $x$ is said to be $\tau$-measurable if and only if, for every $\varepsilon>0$, there exists a projection $e \in \mathscr{M}^{p}$ for which $e(H) \subseteq D(x)$ and $\tau(1-e)<\varepsilon$. We denote by $\widetilde{\mathscr{M}}$ the set of all $\tau$-measurable operators, and with sum and product defined as the respective closures of the algebraic sum and product, the set $\widetilde{\mathscr{M}}$ is a $*$-algebra. The sets $N(\varepsilon, \delta) \quad(\varepsilon, \delta>0)$ consisting of all $x \in \widetilde{\mathscr{M}}$ for which there exists a projection $e \in \mathscr{M}^{p}$ with $e(H) \subseteq D(x),\|x e\| \leq \varepsilon$ and $\tau(1-e) \leq \delta$ form a base at 0 for a metrizable Hausdorff topology in $\widetilde{\mathscr{M}}$ called the measure topology, and equipped with the measure topology, $\widetilde{\mathscr{M}}$ is a complete topological $*$-algebra. The linear subspace $E \subseteq H$ is called $\tau$-dense if for each $\delta>0$, there exists a projection $e \in \mathscr{M}^{p}$ for which $e(H) \subseteq E$ and $\tau(1-e)<\delta$. If $x \in \widetilde{\mathscr{M}}$ and if $E \subseteq D(x)$ is $\tau$-dense, then $x$ is the closure of its restriction to $E$. For these facts, together with their proofs, we refer to the papers of Nelson [Ne] and Terp [Te]. By way of example, we mention first that if $\mathscr{M}$ is $\mathscr{L}(H)$, the von Neumann algebra of all bounded linear operators in $H$ equipped with the usual standard trace, then $\mathscr{M}$ coincides with $\widetilde{\mathscr{M}}$ and in this case the measure topology coincides with the operator norm topology. On the other hand, if $\tau(1)<\infty$, then $\widetilde{\mathscr{M}}$ consists of all densely defined closed linear operators affiliated with $\mathscr{M}$. If $\mathscr{M}$ is commutative then $\mathscr{M}$ may be identified with $L^{\infty}(\Omega, \nu)$ and $\tau(f)=\int_{\Omega} f d \nu$ where $(\Omega, \nu)$ is a localizable measure space. In this case, $\widetilde{\mathscr{M}}$ is the space $S_{0}(\Omega)$ consisting of those measurable complex functions on $\Omega$ which are bounded except on a set of finite measure and the measure topology on $\widetilde{\mathscr{M}}$ may be identified simply with the familiar topology of convergence in measure.

If $x$ is any selfadjoint operator in $H$ and if $x=\int_{(-\infty, \infty)} s d e_{s}^{x}$ is its spectral representation, we will write $\chi_{B}(x)$ for the spectral projection $\int_{B} s d e_{s}^{x}$ whenever $B \subseteq \mathbb{R}$ is a Borel subset. In particular we note that $e_{s}^{x}=\chi_{(-\infty, s]}(x)$. If $x$ is a closed, densely defined linear operator affiliated with $\mathscr{M}$, then the spectral resolution $\chi \cdot(|x|)$ is contained in $\mathscr{M}$, and $x \in \widetilde{\mathscr{M}}$ if and only if there exists $s \in(-\infty, \infty)$ such that $\tau\left(\chi_{(s, \infty)}(|x|)\right)<\infty$. If $x \in \widetilde{\mathscr{M}}$, the function $\mu .(x):[0, \infty) \rightarrow[0, \infty]$ defined by setting

$$
\mu_{t}(x)=\inf \left\{s \geq 0: \tau\left(\chi_{(s, \infty)}(|x|)\right) \leq t\right\}, \quad t \geq 0,
$$

is called the generalized singular value function or decreasing rearrangement of $x$. For basic properties of generalized singular value functions in the present 
setting, we refer to the paper of Fack and Kosaki [FK]. It is useful to note, and easily verified, that a basis of neighbourhoods at zero for the measure topology is given by the sets

$$
M_{\varepsilon, \delta}=\left\{x \in \widetilde{\mathscr{M}}: \mu_{\delta}(x)<\varepsilon\right\}, \quad \varepsilon, \delta>0 .
$$

We remark (cf. [FK, Lemma 3.4]) that if $\left\{x_{n}\right\}$ is a sequence in $\widetilde{\mathscr{M}}$ and if $x_{n} \rightarrow x \in \widetilde{\mathscr{M}}$ for the measure topology then $\mu_{t}\left(x_{n}\right) \rightarrow \mu_{t}(x)$ whenever $t>0$ is a point of continuity of $\mu(x)$. In particular $\mu\left(x_{n}\right) \rightarrow \mu(x)$ a.e. (with respect to Lebesgue measure) on $\mathbb{R}^{+}$. We now gather some remarks concerning the order structure of the space $\widetilde{\mathscr{M}}$.

If $x$ is a selfadjoint linear operator in $H$ and if $($,$) denotes the inner$ product in $H$ then we write as usual

$$
x \geq 0 \text { if and only if }(x \xi, \xi) \geq 0, \quad \text { for all } \xi \in D(x) .
$$

If $x \in \widetilde{\mathscr{M}}$ and if $E \subseteq D(x)$ is $\tau$-dense then it follows that

$$
x \geq 0 \text { if and only if }(x \xi, \xi) \geq 0 \text { for all } \xi \in E,
$$

since $x$ is the closure of its restriction to $E$. It now follows that $\widetilde{\mathscr{M}}$ is an ordered vector space with respect to the partial ordering defined by setting

$$
x \geq y \text { if and only if } x-y \geq 0 \text {. }
$$

Further, if $0 \leq x \in \widetilde{\mathscr{M}}$ then also $y^{*} x y \geq 0$ for all $y \in \widetilde{\mathscr{M}}$.

If $0 \leq x$ is a selfadjoint operator in $H$, then following [Ka], we denote by $q^{x}$ the associated sesquilinear form. The domain $D\left(q^{x}\right)$ of the form $q^{x}$ is $D\left(x^{1 / 2}\right)$ and

$$
q^{x}(\xi, \eta)=\left(x^{1 / 2} \xi, x^{1 / 2} \eta\right), \quad \xi, \eta \in D\left(x^{1 / 2}\right) .
$$

It is not difficult to show that if $0 \leq x, y \in \widetilde{\mathscr{M}}$ then $0 \leq x \leq y$ holds if and only if $q^{x} \leq q^{y}$ holds in the sense of quadratic forms i.e.,

$$
D\left(y^{1 / 2}\right) \subseteq D\left(x^{1 / 2}\right) \text { and }\left\|x^{1 / 2} \xi\right\| \leq\left\|y^{1 / 2} \xi\right\|, \quad \xi \in D\left(y^{1 / 2}\right) .
$$

We now show that the ordered vector space $(\widetilde{\mathscr{M}}, \leq)$ is in fact order complete in the sense of the following.

Proposition 1.1. If $0 \leq x_{\alpha} \uparrow_{\alpha} \leq z$ holds in $\widetilde{\mathscr{M}}$ then $x=\sup _{\alpha} x_{\alpha}$ exists in $\widetilde{\mathscr{M}}$. Proof. Define the form $q$ by setting

$$
D(q)=\left\{\xi: \sup _{\alpha}\left\|x_{\alpha}^{1 / 2} \xi\right\|<\infty\right\}, \quad q(\xi, \xi)=\sup _{\alpha}\left\|x_{\alpha}^{1 / 2} \xi\right\|^{2}, \quad \xi \in D(q)
$$

and

$q(\xi, \eta)=\frac{1}{4}(q(\xi+\eta, \xi+\eta)-q(\xi-\eta, \xi-\eta)+i q(\xi+i \eta, \xi+i \eta)-i q(\xi-i \eta, \xi-i \eta))$ for $\xi, \eta \in D(q)$. Since $\left\|x_{\alpha}^{1 / 2} \xi\right\| \uparrow_{\alpha}$, it is simple to check that

(i) $q(\xi+\eta, \xi+\eta)+q(\xi-\eta, \xi-\eta)=2 q(\xi, \xi)+2 q(\eta, \eta)$,

(ii) $q(\lambda \xi, \lambda \xi)=|\lambda|^{2} q(\xi, \xi)$,

for all $\xi, \eta \in D(q)$ and for all complex numbers $\lambda$ and so by [St, Lemma 6.1] it follows that $q$ is a symmetric nonnegative form in the sense of [Ka] which 
is densely defined since $D(q) \supseteq D\left(z^{1 / 2}\right)$. A standard argument shows that $q$ is closed in the sense of [Ka] and consequently, it follows from [Ka, Theorems VI 2.1 and VI 2.23] that there exists a selfadjoint operator $0 \leq x$ such that $D\left(x^{1 / 2}\right)=D(q)$ and

$$
q(\xi, \eta)=\left(x^{1 / 2} \xi, x^{1 / 2} \eta\right), \quad \xi, \eta \in D(q) .
$$

If now $u \in \mathscr{M}^{\prime}$ is unitary, it follows from the equality $\left\|x_{\alpha}^{1 / 2} \xi\right\|=\left\|x_{\alpha}^{1 / 2} u \xi\right\|$ for all $\alpha$, that $\xi \in D\left(x^{1 / 2}\right)$ if and only if $u \xi \in D\left(x^{1 / 2}\right)$, in which case $q(\xi, \xi)=$ $q(u \xi, u \xi)$. Hence

$$
\left(x^{1 / 2} u \xi, x^{1 / 2} u \eta\right)=(u x \xi, u \eta), \quad \xi \in D(x), \eta \in D\left(x^{1 / 2}\right),
$$

and consequently $\xi \in D(x)$ implies $x^{1 / 2} u \xi \in D\left(x^{1 / 2}\right)$ and $x^{1 / 2} x^{1 / 2} u \xi=u x \xi$. Thus $x \subseteq u^{*} x u$ and a similar argument shows that $u^{*} x u \subseteq x$. Thus $x$ is affiliated with $\mathscr{M}$ and so $x^{1 / 2}$ is affiliated with $\mathscr{M}$. Since $D\left(z^{1 / 2}\right) \subseteq D\left(x^{1 / 2}\right)$ and $D\left(z^{1 / 2}\right)$ is $\tau$-dense it now follows that $x^{1 / 2} \in \widetilde{\mathscr{M}}$ and hence $x \in \widetilde{\mathscr{M}}$. The definition of $x$ now shows that if $z_{0} \in \widetilde{\mathscr{M}}$ and if $z_{0} \geq x_{\alpha}$ for all $\alpha$, then $D\left(z_{0}^{1 / 2}\right) \subseteq D\left(x^{1 / 2}\right)$ and $q^{x}(\xi, \xi) \leq q^{z_{0}}(\xi, \xi)$ holds for all $\xi \in \mathscr{D}\left(z_{0}^{1 / 2}\right)$. This implies that $0 \leq x \leq z_{0}$ and so $x=\sup _{\alpha} x_{\alpha}$ holds in $\widetilde{\mathscr{M}}$.

The referee has pointed out that the previous Proposition 1.1 has been obtained independently by $\mathrm{K}$. Watanabe, of Niigata University.

The following remark is a consequence of the proof of Proposition 1.1.

Corollary 1.2. If $0 \leq x_{\alpha} \uparrow_{\alpha} \leq x$ holds in $\widetilde{\mathscr{M}}$ then $x=\sup _{\alpha} x_{\alpha}$ if and only if

$$
D\left(x^{1 / 2}\right)=\left\{\xi: \sup _{\alpha}\left\|x_{\alpha}^{1 / 2} \xi\right\|<\infty\right\}
$$

and

$$
\left\|x^{1 / 2} \xi\right\|=\sup _{\alpha}\left\|x_{\alpha}^{1 / 2} \xi\right\|, \quad \xi \in D\left(x^{1 / 2}\right) .
$$

Proposition 1.3. If $0 \leq x_{\alpha} \uparrow_{\alpha} x$ holds in $\widetilde{\mathscr{M}}$ then $0 \leq y^{*} x_{\alpha} y \uparrow_{\alpha} y^{*} x y$ holds in $\widetilde{\mathscr{M}}$ for all $y \in \widetilde{\mathscr{M}}$.

Proof. If $y \in \widetilde{\mathscr{M}}$ then it is clear that $0 \leq y^{*} x_{\alpha} y \uparrow_{\alpha} \leq y^{*} x y$ holds in $\widetilde{\mathscr{M}}$, so that if $z=\sup _{\alpha} y^{*} x_{\alpha} y$ then $z \in \widetilde{\mathscr{M}}$ and $0 \leq z \leq y^{*} x y$. From the preceding corollary, it follows that

$$
\left\|\left(y^{*} x_{\alpha} y\right)^{1 / 2} \xi\right\| \uparrow_{\alpha}\left\|z^{1 / 2} \xi\right\|, \quad \xi \in D\left(z^{1 / 2}\right),
$$

and

$$
\left\|x_{\alpha}^{1 / 2}(y \xi)\right\| \uparrow_{\alpha}\left\|x^{1 / 2} y \xi\right\|
$$

for all $\xi \in D\left(x^{1 / 2} y\right)=D\left(\left|x^{1 / 2} y\right|\right)=D\left(\left(y^{*} x y\right)^{1 / 2}\right) \subseteq D\left(z^{1 / 2}\right)$. Since

$$
\left\|\left(y^{*} x_{\alpha} y\right)^{1 / 2} \xi\right\|^{2}=\left\|\left|x_{\alpha}^{1 / 2} y\right| \xi\right\|^{2}=\left\|x_{\alpha}^{1 / 2} y \xi\right\|^{2}
$$

for all $\alpha$ and all $\xi \in D\left(\left(y^{*} x_{\alpha} y\right)^{1 / 2}\right)=\mathscr{D}\left(x_{\alpha}^{1 / 2} y\right)$ it follows that $\left\|z^{1 / 2} \xi\right\|^{2}=$ $\left\|x^{1 / 2} y \xi\right\|^{2}$ holds for all $\xi \in D\left(\left(y^{*} x y\right)^{1 / 2}\right)$ and so $(z \xi, \xi)=\left(y^{*} x y \xi, \xi\right)$ for all $\xi \in D\left(y^{*} x y\right)$. It now follows that $z=y^{*} x y$ since $D\left(y^{*} x y\right)$ is $\tau$-dense.

Proposition 1.4. The positive cone $\widetilde{\mathscr{M}}_{+}$is closed for the measure topology.

Proof. If $x \in \widetilde{\mathscr{M}}$ and $x$ belongs to the closure in $\widetilde{\mathscr{M}}$ of $\widetilde{\mathscr{M}_{+}}$for the measure topology then certainly $x=x^{*}$ by the continuity of the adjoint operation on 
for the measure topology. There exists a projection $e \in \mathscr{M}^{p}$ such that $x^{-}=-e x e$. If $\varepsilon>0, \delta>0$ are given, there exists $y \in \widetilde{\mathscr{M}}_{+}$such that $\mu_{\delta}(x-y)<\varepsilon$. Since $0 \leq x^{-} \leq e(y-x) e$, it follows that $\mu_{\delta}\left(x^{-}\right)<\varepsilon$ for all $\varepsilon>0, \delta>0$ and this implies that $x^{-}=0$.

As usual, we denote by $L^{0}\left(\mathbb{R}^{+}\right)$the space of all complex-valued Lebesgue measurable functions on the half line $\mathbb{R}^{+}$with the usual identifications.

Lemma 1.5. If $0 \leq x \in \widetilde{\mathscr{M}}$ then

$$
\mu(x)=\sup \left\{\mu(\text { exe }): e \in \mathscr{M}^{p}, \tau(e)<\infty \text { and exe } \in \mathscr{M}\right\},
$$

where the right-hand supremum is taken in $L^{0}\left(\mathbb{R}^{+}\right)$.

Proof. We denote by $w$ the supremum on the right-hand side. Since $\mu($ exe $) \leq$ $\mu(x)$ holds for every projection $e \in \mathscr{M}^{p}$ by [FK, Lemma 2.5(vi)], we have $w \leq \mu(x)$. Set $\alpha_{\infty}=\lim _{t \rightarrow \infty} \mu_{t}(x)$. We first show that $w \geq \alpha_{\infty} 1$. It may clearly be assumed that $\alpha_{\infty}>0$. If $0<\alpha<\alpha_{\infty}$, then since $\tau\left(\chi_{(\alpha, \beta]}(x)\right) \rightarrow \infty$ as $\beta \rightarrow \infty$, for any natural number $n$, there exists a projection $e \in \mathscr{M}^{p}$ and a number $\beta_{0}>\alpha$ such that $n \leq \tau(e)<\infty$ and $e \leq \chi_{\left(\alpha, \beta_{0}\right]}(x)$. Since $\alpha e \leq$ exe $\leq \beta_{0} e$, it follows that exe $\in \mathscr{M}$ and $\alpha \chi_{[0, n)} \leq \alpha \chi_{[0, \tau(e))} \leq \mu($ exe $) \leq w$, and it is now clear that $w \geq \alpha_{\infty} 1$. If we set

$$
t_{0}=\inf \left\{t \geq 0: \mu_{t}(x)=\alpha_{\infty}\right\},
$$

then we have $w \geq \chi_{\left[t_{0}, \infty\right)} \mu(x)$. Suppose that $0<t<t_{0}$. There exists $s>t$ such that $\mu_{s}(x)<\mu_{t}(x)$. If $\gamma=\mu_{s}(x)$, then $t \leq \tau\left(\chi_{(\gamma, \infty)}(x)\right) \leq s$. Let $\delta>\gamma$ and set $e=\chi_{(\gamma, \delta]}(x)$. It then follows that exe $\in \mathscr{M}$ and so

$$
w(r) \geq \mu_{r}(\text { exe })=\chi_{[0, \tau(e))} \mu_{r+\eta}(x), \quad r \text {-a.e. },
$$

where $\eta=\tau\left(\chi_{(\delta, \infty)}(x)\right)$. If now $\delta \rightarrow \infty$, it follows that $\eta \nrightarrow 0$ and so

$$
w \geq \chi_{\left[0, \tau\left(\chi_{(y, \infty)}(x)\right)\right)} \mu(x) \geq \chi_{[0, t)} \mu(x),
$$

hence $w \geq \chi_{\left[0, t_{0}\right)} \mu(x)$, and this clearly suffices to complete the proof. 2.7].

It is now convenient to recall the following special case of [FK, Proposition

Lemma 1.6. If $0 \leq x \in \mathscr{M}$, then $\tau(x)=\int_{[0, \infty)} \mu_{t}(x) d t$ holds in the sense that if either side is finite then so is the other, in which case equality holds.

Proposition 1.7. If $0 \leq x_{\alpha} \uparrow_{\alpha} x$ holds in $\widetilde{\mathscr{M}}$ then $\mu_{t}\left(x_{\alpha}\right) \uparrow_{\alpha} \mu_{t}(x)$ holds for all $t \geq 0$.

Proof. If $e \in \mathscr{M}^{p}$ is such that $\tau(e)<\infty$ and exe $\in \mathscr{M}$ then $0 \leq e x_{\alpha} e \uparrow_{\alpha}$ exe holds in $\mathscr{M}$ and consequently $0 \leq \mu\left(e_{\alpha} e\right) \uparrow_{\alpha} \mu(e x e)$ holds in $L^{1}\left(\mathbb{R}^{+}\right)$since

$$
\int_{[0, \infty)} \mu_{t}\left(e x_{\alpha} e\right) d t=\tau\left(e x_{\alpha} e\right) \uparrow_{\alpha} \tau(\text { exe })=\int_{[0, \infty)} \mu_{t}(\text { exe }) d t
$$

which follows from the preceding Lemma 1.6. The statement of the proposition now follows from Lemma 1.5 , and the right continuity of $\mu(x)$.

The final result of this section is a consequence of the argument of [Di, I.3.4, Corollary 5]. 
Proposition 1.8. If $0 \leq x \in \widetilde{\mathscr{M}}$, then there exists $\left\{x_{\alpha}\right\} \subseteq \mathscr{M}$ with $\tau\left(x_{\alpha}\right)<\infty$ for each index $\alpha$ and such that $0 \leq x_{\alpha} \uparrow_{\alpha} x$ holds in $\widetilde{\mathscr{M}}$.

\section{REARRANGEMENT INVARIANT SPACES OF MEASURABLE OPERATORS}

In this section we gather some basic properties of normed spaces of measurable operators which are the natural analogues to the rearrangement invariant function spaces studied by Luxemburg [Lu] and the symmetric spaces on the half-line $[0, \infty)$ of Krein, Petunin, and Semenov [KPS]. We begin with the following definition.

Definition 2.1. A normed linear subspace $E \subseteq \widetilde{\mathscr{M}}$ is called a (normed) rearrangement invariant operator space if and only if whenever $x \in \widetilde{\mathscr{M}}, y \in E$ satisfy $\mu(x) \leq \mu(y)$ it follows that $x \in E$ and $\|x\|_{E} \leq\|y\|_{E}$.

If $E \subseteq \widetilde{\mathscr{M}}$ is a normed rearrangement invariant operator space then it is a simple consequence of the definition that

(i) $x \in E \Leftrightarrow x^{*} \in E \Leftrightarrow|x| \in E$; and $\|x\|_{E}=\left\|x^{*}\right\|_{E}=\||x|\|_{E}$,

(ii) $x \in E, 0 \leq u, v \in \mathscr{M}$ imply that $u x v \in E$ and

$$
\|u x v\|_{E} \leq\|u\|_{\infty}\|v\|_{\infty}\|x\|_{E}
$$

Proposition 2.2. If $E \subseteq \widetilde{\mathscr{M}}$ is a normed rearrangement invariant operator space then the natural inclusion of $E$ into $\widetilde{\mathscr{M}}$ is continuous.

Proof. Let $\varepsilon>0$ be given. We first show that there exists a constant $C=$ $C(\varepsilon)>0$ such that

$$
e \in E \cap \mathscr{M}^{p} \text { and } \tau(e)>\varepsilon \Rightarrow\|e\|_{E}>C
$$

Indeed, let

$$
m=\inf \left\{\tau(e): 0 \neq e \in \mathscr{M}^{p}\right\} .
$$

First suppose that $m=0$. There exists $0 \neq f \in \mathscr{M}^{p}$ such that $\tau(f)<\varepsilon$. If $e \in E \cap \mathscr{M}^{p}$ with $\tau(e)>\varepsilon$, then $\tau(f)<\tau(e)$ and so $\|f\|_{E} \leq\|e\|_{E}$; we can take $C=\frac{1}{2}\|f\|_{E}$. Now suppose $m>0$. We show in this case that there exists $C>0$, independent of $\varepsilon$, such that

$$
\|e\|_{E}>C, \quad \text { for all } e \in E \cap \mathscr{M}^{p} \text {. }
$$

If this is not true, then there exists a sequence $\left\{e_{n}\right\} \subseteq E \cap \mathscr{M}^{p}$ such that $\left\|e_{n}\right\|_{E} \rightarrow_{n} 0$. In this case, each projection dominates a minimal projection of finite trace and so we may assume that each $e_{n}$ is minimal and of finite trace. It follows that

$$
e_{i} \wedge e_{j}=0 \text { for } i \neq j
$$

Choose $l \in \mathbb{N}$ such that $l m \geq \tau\left(e_{1}\right)$. For each $k \in \mathbb{N}$, we have

$$
\tau\left(e_{k+1} \vee \cdots \vee e_{k+l}\right)=\tau\left(e_{k+1}\right)+\cdots+\tau\left(e_{k+l}\right) \geq l m \geq \tau\left(e_{1}\right) .
$$

It follows that

$$
0<\left\|e_{1}\right\|_{E} \leq\left\|e_{k+1} \vee \cdots \vee e_{k+l}\right\|_{E} \leq\left\|e_{k+1}\right\|_{E}+\cdots+\left\|e_{k+l}\right\|_{E}, \quad \text { for all } k \in \mathbb{N},
$$

which is a contradiction to the assumption that $\left\|e_{n}\right\|_{E} \rightarrow_{n} 0$. Now let $0 \leq$ $x \in E$ be such that $\|x\|_{E} \leq \varepsilon C$. We may assume that $\mu_{\varepsilon}(x)>0$. If $\alpha$ 
is such that $0<\alpha<\mu_{\varepsilon}(x)$, then $\varepsilon<\tau\left(\chi_{(\alpha, \infty)}(x)\right)$. From the inequalities $0 \leq \alpha \chi_{(\alpha, \infty)}(x) \leq x \chi_{(\alpha, \infty)}(x)$, and the assertion in the first part of the proof, we obtain that $\alpha C<\alpha\left\|\chi_{(\alpha, \infty)}(x)\right\|_{E} \leq\|x\|_{E} \leq \varepsilon C$. It now follows also that $\mu_{\varepsilon}(x) \leq \varepsilon$ and this suffices to complete the proof.

We remark that the preceding proposition is a simple extension of [DDP1 Lemma 4.4].

Before proceeding, we note the following simple criterion for a normed rearrangement invariant operator space to be a Banach space.

Proposition 2.3. A normed rearrangement invariant operator space $E \subseteq \widetilde{\mathscr{M}}$ is a Banach space if and only if each increasing Cauchy sequence in $E$ is convergent.

Proof. The stated condition is clearly necessary. Conversely, suppose that $\left\{x_{n}\right\}_{n=1}^{\infty} \subseteq E$ is a sequence of selfadjoint elements for which

$$
\sum_{n=1}^{\infty}\left\|x_{n+1}-x_{n}\right\|_{E}<\infty
$$

Setting

$$
u_{n}=\sum_{i=1}^{n-1}\left(x_{i+1}-x_{i}\right)^{+}, \quad v_{n}=\sum_{i=1}^{n-1}\left(x_{i+1}-x_{i}\right)^{-}, \quad n=2,3, \ldots,
$$

it is then clear that the sequences $\left\{u_{n}\right\},\left\{v_{n}\right\} \subseteq E$ are increasing Cauchy sequences and hence convergent to $u, v \in E$. It follows that $x_{n} \rightarrow x_{1}+u-v$ and by this the proof is complete.

Corollary 2.4. If $E \subseteq \widetilde{\mathscr{M}}$ is a normed rearrangement invariant operator space with the property

$$
\begin{gathered}
0 \leq x_{n} \uparrow_{n} \subseteq E, \quad \sup _{n}\left\|x_{n}\right\|_{E}<\infty, \\
x=\sup _{n} x_{n} \text { exists in } \widetilde{\mathscr{M}} \Rightarrow x \in E, \quad\|x\|_{E}=\sup _{n}\left\|x_{n}\right\|_{E},
\end{gathered}
$$

then $E$ is a Banach space.

Proof. If $0 \leq x_{n} \uparrow_{n} \subseteq E$ is $\|\cdot\|_{E}$-Cauchy, then the sequence $\left\{x_{n}\right\}$ is Cauchy and hence convergent in the measure topology to some $x \in \widetilde{\mathscr{M}}$. Since the positive cone of $\widetilde{\mathscr{M}}$ is closed for the measure topology, it follows that $x=\sup _{n} x_{n}$ holds in $\widetilde{\mathscr{M}}$. Consequently $0 \leq x \in E$ and the same argument yields that

$$
\left\|x-x_{n}\right\|_{E}=\sup \left\{\left\|x_{m}-x_{n}\right\|_{E}: m>n\right\},
$$

from which it follows that $x_{n} \rightarrow x$ holds in $E$ and the proof is complete.

We remark that the property stated in the above corollary is (essentially) the property referred to by Yeadon [Ye3] as the Fatou property.

If $x, y \in \widetilde{\mathscr{M}}$, we shall say that $x$ is submajorized by $y$, written $x \prec \prec y$, if and only if

$$
\int_{0}^{\alpha} \mu_{t}(x) d t \leq \int_{0}^{\alpha} \mu_{t}(y) d t, \quad \text { for all } \alpha \geq 0
$$


A normed rearrangement invariant operator space $E \subseteq \widetilde{\mathscr{M}}$ will be called symmetric if and only if

$$
x, y \in E \text { and } x \prec \prec y \Rightarrow\|x\|_{E} \leq\|y\|_{E} .
$$

We identify $L^{\infty}\left(\mathbb{R}^{+}\right)$throughout as a commutative von Neumann algebra acting by multiplication on $L^{2}\left(\mathbb{R}^{+}\right)$with trace given by integration with respect to Lebesgue measure. A normed space $E\left(\mathbb{R}^{+}\right)$of almost everywhere finite measurable functions on the half-line $\mathbb{R}^{+}$will be called a rearrangement invariant (symmetric) function space on $\mathbb{R}^{+}$if the corresponding conditions hold with respect to the von Neumann algebra $L^{\infty}\left(\mathbb{R}^{+}\right)$. If $E\left(\mathbb{R}^{+}\right)$is a rearrangement invariant normed function space on $\mathbb{R}^{+}$which is a Banach space in its given norm, then $E\left(\mathbb{R}^{+}\right)$will be called a rearrangement invariant Banach function space.

If $E\left(\mathbb{R}^{+}\right)$is a normed rearrangement invariant function space on $\mathbb{R}^{+}$, we set

$$
E(\mathscr{M})=\left\{x \in \widetilde{\mathscr{M}}: \mu(x) \in E\left(\mathbb{R}^{+}\right)\right\},
$$

and if $x \in E(\mathscr{M})$, we define $\|x\|_{E(\mathscr{M})}=\|\mu(x)\|_{E\left(\mathbf{R}^{+}\right)}$. If, in addition $E\left(\mathbb{R}^{+}\right)$is symmetric, then it can be shown that $E(\mathscr{M})$ is a normed, symmetric, rearrangement invariant operator space which is a Banach space if $E\left(\mathbb{R}^{+}\right)$is a Banach space (see [DDP1, DDP3]).

If $x \in \widetilde{\mathscr{M}}$ then it is a simple consequence of [FK, Proposition 2.2] that $x \in \mathscr{M}$ if and only if $\mu(x) \in L^{\infty}\left(\mathbb{R}^{+}\right)$and in this case $\|\mu(x)\|_{\infty}=\|x\|_{\infty}$. It follows simply that the equalities

$$
L^{\infty}(\mathscr{M})=\mathscr{M}, \quad\left(L^{1} \cap L^{\infty}\right)(\mathscr{M})=L^{1}(\mathscr{M}) \cap \mathscr{M},
$$

hold in the sense of Banach spaces. It should also be observed, via Lemma 1.6, that $L^{1}(\mathscr{M}) \cap \mathscr{M}$ coincides with the two-sided ideal introduced in [Di, Proposition I.6.1].

The following result is essentially proved in [FK], remark after Theorem 4.4 and is stated in [Ov2, Theorem 3]. See also [PS].

Proposition 2.5. If $x \in \widetilde{\mathscr{M}}$ and if $t>0$, then

$$
\int_{[0, t]} \mu_{s}(x) d s=\inf \left\{\|y\|_{1}+t\|z\|_{\infty}: x=y+z, y \in L^{1}(\mathscr{M}), z \in \mathscr{M}\right\} .
$$

An immediate consequence of the preceding Proposition 2.5 is the Banach space equality $\left(L^{1}+L^{\infty}\right)(\mathscr{M})=L^{1}(\mathscr{M})+\mathscr{M}$.

It follows immediately from [KPS, Theorem II4.1] that if $E\left(\mathbb{R}^{+}\right)$is a symmetric rearrangement invariant Banach function space on $\mathbb{R}^{+}$, then the Banach space $E(\mathscr{M})$ is intermediate for the Banach couple $\left(L^{1}(\mathscr{M}), \mathscr{M}\right)$, in the sense of [KPS, Chapter I].

For notational convenience, we will write $G(\mathscr{M}), H(\mathscr{M})$ respectively for the operator spaces $L^{1}(\mathscr{M})+\mathscr{M}, L^{1}(\mathscr{M}) \cap \mathscr{M}$.

Proposition 2.6. If $x \in \widetilde{\mathscr{M}}$ then the following statements are equivalent.

(a) $x \in G(\mathscr{M})$.

(b) $\int_{[0, a)} \mu_{t}(x) d t<\infty$ for some $a>0$.

(c) $\int_{[0, a)} \mu_{t}(x) d t<\infty$ for all $a>0$. 
(d) $e|x| e \in L^{1}(\mathscr{M})$ for all projections $e \in H(\mathscr{M})$.

Proof. It suffices to prove the equivalence (a) $\Leftrightarrow$ (d). If $x \in G(\mathscr{M})$ and $e \in$ $H(\mathscr{M})$, then from the inequality $\mu(e|x| e) \leq \chi_{[0, \tau(e))} \mu(x)$ it follows immediately that $\mu(e|x| e) \in L^{1}\left(\mathbb{R}^{+}\right)$.

Conversely, suppose that $x \in \widetilde{\mathscr{M}}$ and that $e|x| e \in L^{1}(\mathscr{M})$ for all projections $e \in H(\mathscr{M})$. If $x \in \mathscr{M}$, there is nothing to prove. We may therefore assume that there exists $\alpha>0$ such that $0<\tau\left(\chi_{(\alpha, \infty)}(|x|)\right)<\infty$. Setting $e=\chi_{(\alpha, \infty)}(|x|)$, it follows that $0<\tau(e)<\infty$ and $\mu(e|x| e)=\chi_{[0, \tau(e))} \mu(x)$. It follows immediately that $\int_{[0, \tau(e)]} \mu_{t}(x) d t<\infty$, and consequently $x \in G(\mathscr{K})$ and the proof is complete.

We define

$$
\widetilde{\mathscr{M}_{0}}=\left\{x \in \widetilde{\mathscr{M}}: \mu_{t}(x) \rightarrow 0 \text { as } t \rightarrow \infty\right\} .
$$

Observe that $\widetilde{\mathscr{M}_{0}}$ is a linear subspace of $\widetilde{\mathscr{M}}$ which is solid in the sense that if $x \in \widetilde{\mathscr{M}}_{0}$ and if $y \in \widetilde{\mathscr{M}}$ and $\mu(y) \leq \mu(x)$ then also $y \in \widetilde{\mathscr{M}}_{0}$. It is clear that $x \in \widetilde{\mathscr{M}}_{0}$ if and only if $\tau\left(\chi_{(s, \infty)}(|x|)\right)<\infty$ for all $s>0$. Moreover it is clear that $H(\mathscr{M}) \subseteq \widetilde{\mathscr{M}}_{0}$. It is worth noting that if $\mathscr{M}$ is $\mathscr{L}(H)$ with standard trace, then $\widetilde{\mathscr{M}}_{0}$ is precisely the ideal of compact operators.

Proposition 2.7. (a) $\widetilde{\mathscr{M}_{0}}$ is the closure of $H(\mathscr{M})$ in $\widetilde{\mathscr{M}}$ for the measure topology.

(b) The closure of $H(\mathscr{M})$ in the space $G(\mathscr{M})$ is $\widetilde{\mathscr{M}}_{0} \cap G(\mathscr{M})$.

Proof. (a) Let $0 \leq x \in \widetilde{\mathscr{M}}_{0}$ and set

$$
x_{n}=x \chi_{(1 / n, n]}(x), \quad n=1,2, \ldots
$$

It is clear that $x_{n} \in H(\mathscr{M})$ and that $0 \leq x_{n} \leq x, n=1,2, \ldots$. Since

$$
\left\|\left(x-x_{n}\right) \chi_{(-\infty, n]}(x)\right\|_{\infty} \leq \frac{1}{n}, \quad n=1,2, \ldots,
$$

and since $\tau\left(1-\chi_{(-\infty, n]}(x)\right) \rightarrow 0$ as $n \rightarrow \infty$, it follows that $x_{n} \rightarrow x$ for the measure topology.

Conversely, suppose that $x$ belongs to the closure of $H(\mathscr{M})$ for the measure topology. If $\varepsilon>0$ is given, there exists $y \in H(\mathscr{K})$ such that $\mu_{\varepsilon}(x-y) \leq \varepsilon$, and so $\mu_{t+\varepsilon}(x) \leq \mu_{\varepsilon}(x-y)+\mu_{t}(y) \leq \varepsilon+\mu_{t}(y)$ hold for each $t, \varepsilon>0$. Consequently $\lim _{t \rightarrow \infty} \mu_{t}(x) \leq \varepsilon$.

(b) If $x \in G(\mathscr{M})$ belongs to the closure of $H(\mathscr{M})$ in $G(\mathscr{M})$, then $x$ belongs to the closure of $H(\mathscr{M})$ in $\widetilde{\mathscr{M}}$ for the measure topology by Proposition 2.2 and consequently $x \in \widetilde{\mathscr{K}}_{0}$ by part (a) preceding. Conversely, if $0 \leq x \in \widetilde{\mathscr{K}}_{0} \cap G(\mathscr{M})$, set

$$
x_{n}=x \chi_{(1 / n, n]}(x), \quad n=1,2, \ldots,
$$

and note that $x_{n} \in H(\mathscr{M}), n=1,2, \ldots$. Since

$$
\mu_{t}\left(x \chi_{(n, \infty)}(x)\right)=\chi_{\left[0, \tau\left(\chi_{(n, \infty)}(x)\right)\right)}(t) \mu_{t}(x),
$$


we have $x \chi_{(n, \infty)}(x) \in L^{1}(\mathscr{M})$ and so

$$
\begin{aligned}
\left\|x-x_{n}\right\|_{G(\mathscr{K})} & =\left\|x \chi_{(n, \infty)}(x)+x \chi_{[0,1 / n]}(x)\right\|_{G(\mathscr{M})} \\
& \leq\left\|x \chi_{(n, \infty)}(x)\right\|_{L^{1}(\mathscr{M})}+\left\|x \chi_{[0,1 / n]}(x)\right\|_{\mathscr{M}} \\
& \leq \int_{0}^{\tau\left(\chi_{(n, \infty)}(x)\right)} \mu_{t}(x) d t+\frac{1}{n} \\
& \rightarrow 0 \quad \text { as } n \rightarrow \infty .
\end{aligned}
$$

The rearrangement invariant Banach function space $E\left(\mathbb{R}^{+}\right)$is called minimal if and only if $H\left(\mathbb{R}^{+}\right)$is dense in $E\left(\mathbb{R}^{+}\right)$. We remark [KPS, II $\S 4.5$ ] that $E\left(\mathbb{R}^{+}\right)$ is minimal if and only if

$$
\left\|f \chi_{(n, \infty)}\right\|_{E} \rightarrow 0 \text { and }\left\|(|f|-n 1)^{+}\right\|_{E} \rightarrow 0 \text {, }
$$

as $n \rightarrow \infty$ for each $f \in E\left(\mathbb{R}^{+}\right)$. Further, if $E\left(\mathbb{R}^{+}\right)$is minimal then $\mu_{t}(|f|) \rightarrow 0$ as $t \rightarrow \infty$, for each $f \in E\left(\mathbb{R}^{+}\right)$.

Proposition 2.8. If the symmetric rearrangement invariant Banach function space $E\left(\mathbb{R}^{+}\right)$is minimal, then $H(\mathscr{M})$ is dense in $E(\mathscr{M})$.

Proof. Let $0 \leq x \in E(\mathscr{M})$ and set

$$
x_{n}=x \chi_{(1 / n, n]}(x), \quad n=1,2, \ldots .
$$

Note that $0 \leq x_{n} \leq n \chi_{(1 / n, \infty)}(x)$, and since $x \in \widetilde{\mathscr{M}}_{0}$ by the minimality of $E\left(\mathbb{R}^{+}\right)$, it follows also that $\tau\left(\chi_{(1 / n, \infty)}(x)\right)<\infty$, and so $x_{n} \in H(\mathscr{M})$, for $n=$ $1,2, \ldots$. Consequently,

$$
\begin{aligned}
\left\|x-x_{n}\right\|_{E(\mathscr{M})} & \leq\|x-x \wedge n\|_{E(\mathscr{M})}+\left\|x \wedge n-x_{n}\right\|_{E(\mathscr{K})} \\
& \leq\left\|(x-n)^{+}\right\|_{E(\mathscr{M})}+\left\|x \chi_{[0,1 / n]}(x)\right\|_{E(\mathscr{M})} \\
& =\left\|\left(\mu_{t}(x)-n\right)^{+}\right\|_{E}+\left\|\chi_{\left(\tau\left(\chi_{(1 / n, \infty)}(x)\right), \infty\right)}(t) \mu_{t}(x)\right\|_{E} \\
& \rightarrow 0 \text { as } n \rightarrow 0,
\end{aligned}
$$

by minimality of $E\left(\mathbb{R}^{+}\right)$.

\section{EXTENSION OF THE TRACE TO $\widetilde{\mathscr{M}_{+}}$}

To formulate a natural extension of the well-known dominated convergence theorem, it is desirable to extend the trace $\tau$ to the positive cone of $\widetilde{\mathscr{M}}$. See, for example, [FK, Theorem 3.5]. In this section we show that the trace extends naturally to the positive cone $\widetilde{\mathscr{M}}_{+}$so that the basic properties, particularly that of additivity and normality, are preserved. This does not appear to follow in a direct manner from the approach of [FK]. A number of the necessary preliminary results which follow are of course well known and may be found, for example, in [Di].

As in [Di, Proposition I.6.1], we continue to denote by $\tau$ the unique linear extension to $H(\mathscr{M})$ of the restriction of $\tau$ to $H(\mathscr{M})_{+}$. The linear functional $\tau$ is unitarily invariant on $H(\mathscr{M})$ and, as shown in [Di, Theorem I.6.8], if $x \in H(\mathscr{M})$ and $y \in \mathscr{M}$ then

(a) $\tau(x y)=\tau(y x)$, and

(b) $|\tau(x y)| \leq\|y\|_{\infty} \tau(|x|)$.

Since the space $L^{1}\left(\mathbb{R}^{+}\right)$is minimal, it now follows from Proposition 2.8 that 
$L^{1}(\mathscr{M})$ is the completion of $H(\mathscr{M})$ with respect to $\|\cdot\|_{1}$ so that $\tau$ extends uniquely to a bounded linear functional on $L^{1}(\mathscr{M})$, which we again denote by $\tau$. It is not difficult to see that the assertions (a), (b) preceding now continue to hold whenever $x \in L^{1}(\mathscr{M})$ and $y \in \mathscr{M}$. Moreover, if $0 \leq x \in L^{1}(\mathscr{M})$ then the equality

$$
\tau(x)=\int_{[0, \infty)} \mu_{t}(x) d t
$$

continues to hold. In fact, if $x_{n}=x \wedge n 1, n=1,2, \ldots$, then it is clear that $x_{n} \in H(\mathscr{M}), n=1,2, \ldots$, and that

$$
\left\|x-x_{n}\right\|_{1}=\left\|(\mu(x)-n 1)^{+}\right\|_{1} \rightarrow 0 \text { as } n \rightarrow \infty,
$$

and consequently $\tau\left(x_{n}\right) \rightarrow \tau(x)$ as $n \rightarrow \infty$ by continuity. Since

$$
\mu\left(x_{n}\right)=\mu(x) \wedge n 1, \quad n=1,2, \ldots,
$$

it follows that $\mu\left(x_{n}\right) \uparrow_{n} \mu(x)$, and the stated equality now follows from Lemma 1.6.

Lemma 3.1. If $x \in \widetilde{\mathscr{M}}$, then $x^{*} x \in L^{1}(\mathscr{M})$ if and only if $x x^{*} \in L^{1}(\mathscr{M})$, in which case $\tau\left(x^{*} x\right)=\tau\left(x x^{*}\right)$.

Proof. This now follows from the equalities

$$
\mu\left(x^{*} x\right)=\mu\left(|x|^{2}\right)=(\mu(x))^{2}=\left(\mu\left(x^{*}\right)\right)^{2}=\mu\left(x^{*} x\right)
$$

and the above remarks.

Proposition 3.2 (Beppo-Levi property). If $0 \leq x_{\alpha} \uparrow_{\alpha} \subseteq L^{1}(\mathscr{M})$ and if $\sup _{\alpha}\left\|x_{\alpha}\right\|_{1}$ $<\infty$ then $x=\sup _{\alpha} x_{\alpha}$ exists in $L^{1}(\mathscr{M})$ and, $\left\|x-x_{\alpha}\right\|_{1} \downarrow_{\alpha} 0$. Moreover there exists a sequence $\left\{x_{\alpha_{n}}\right\} \subseteq\left\{x_{\alpha}\right\}$ such that $x=\sup _{n} x_{\alpha_{n}}$.

Proof. The conditions $0 \leq x_{\alpha} \uparrow_{\alpha}, \sup _{\alpha} \tau\left(x_{\alpha}\right)<\infty$ imply that the net $\left\{x_{\alpha}\right\}$ is Cauchy in $L^{1}(\mathscr{K})$ and hence convergent to some element $x \in L^{1}(\mathscr{M})$. That $x=\sup _{\alpha} x_{\alpha}$ holds is a simple consequence of Corollary 1.4. By the continuity of $\tau$ it follows that $\tau(x)=\sup _{\alpha} \tau\left(x_{\alpha}\right)$ and this implies that $\left\|x-x_{\alpha}\right\|_{1} \downarrow_{\alpha} 0$. Now $\tau(x)=\sup _{\alpha}\left\|x_{\alpha}\right\|_{1}$, so there exists a sequence $\left\{x_{\alpha_{n}}\right\} \subseteq\left\{x_{\alpha}\right\}$ such that $0 \leq x_{\alpha_{n}} \uparrow_{n}$ and $\sup _{n} \tau\left(x_{\alpha_{n}}\right)=\tau(x)$. Using the first part and the fact that $\tau$ is faithful, it is not difficult to see that $x=\sup _{n} x_{\alpha_{n}}$, and by this the proof is complete.

It is not difficult to prove exact noncommutative extensions of the familiar Fatou's Lemma and the Dominated Convergence Theorem of Lebesgue, which we now state. For the proofs, we refer to [FK, Theorem 3.5]. The reader's attention is also drawn to the alternative approaches given in [St, Le].

Proposition 3.3 (Fatou's Lemma). (i) If $\left\{x_{n}\right\} \subseteq L^{1}(\mathscr{M})$ is such that $x_{n} \rightarrow x$ for the measure topology and if liminf $\left\|x_{n}\right\|_{1}<\infty$ then $x \in L^{1}(\mathscr{M})$ and $\|x\|_{1} \leq$ $\liminf \left\|x_{n}\right\|_{1}$.

(ii) (Dominated Convergence Theorem). If $\left\{x_{n}\right\} \subseteq L^{1}(\mathscr{M})$ is such that $x_{n} \rightarrow$ $x$ for the measure topology and if

$$
\mu\left(x_{n}\right) \leq f \in L^{1}\left(\mathbb{R}^{+}\right), \quad \text { for } n=1,2, \ldots,
$$

then $x \in L^{1}(\mathscr{M})$ and $\left\|x_{n}-x\right\|_{1} \rightarrow 0$.

In the trace ideal setting, a restricted version of the following result may be found in [GK, Theorem III.8.2]. 
Proposition 3.4. If $x, y \in \widetilde{\mathscr{M}}$ and if $x y, y x \in L^{1}(\mathscr{M})$, then $\tau(x y)=\tau(y x)$. If in addition, $0 \leq x \in \widetilde{\mathscr{M}_{0}}$ and $0 \leq y \in \widetilde{\mathscr{M}}$ then $x^{1 / 2} y x^{1 / 2}, y^{1 / 2} x y^{1 / 2} \in L^{1}(\mathscr{M})$ and

$$
\tau(x y)=\tau\left(x^{1 / 2} y x^{1 / 2}\right)=\tau\left(y^{1 / 2} x y^{1 / 2}\right) .
$$

Proof. The first assertion is just [BK, Theorem 17] and we thank the referee for bringing this to our attention. We prove only the second assertion. We assume that $0 \leq x \in \widetilde{\mathscr{M}}_{0}$, that $0 \leq y \in \widetilde{\mathscr{M}}$ and that $x y, y x \in L^{1}(\mathscr{M})$. We set

$$
e=\chi_{(0, \infty)}\left(x^{1 / 2}\right), \quad e_{n}=\chi_{(1 / n, n]}\left(x^{1 / 2}\right), \quad n=1,2, \ldots
$$

We observe that $e_{n} x^{1 / 2}=x^{1 / 2} e_{n} \in H(\mathscr{M})$. As in the proof of Proposition 2.7(a), it follows that $e_{n} x^{1 / 2}=x^{1 / 2} e_{n} \rightarrow x^{1 / 2}$ for the measure topology. We now note that $e_{n} x^{1 / 2} y \in L^{1}(\mathscr{M}), n=1,2, \ldots$ In fact, $\frac{1}{n} e_{n} \leq x^{1 / 2}$ implies that $e_{n} \leq n^{2} x$ and so

$$
\left(e_{n} x^{1 / 2} y\right)^{*}\left(e_{n} x^{1 / 2} y\right)=y x^{1 / 2} e_{n} x^{1 / 2} y \leq n^{2} y x^{2} y=(n x y)^{*}(n x y),
$$

and hence $\left|e_{n} x^{1 / 2} y\right| \leq n|x y| \in L^{1}(\mathscr{M})$.

Now the inequalities

$$
\begin{aligned}
\tau\left(e_{n} x y\right) & =\tau\left(e_{n} x^{1 / 2} e_{n} x^{1 / 2} y\right)=\tau\left(e_{n} x^{1 / 2} y e_{n} x^{1 / 2}\right) \\
& =\tau\left(e_{n} x^{1 / 2} y x^{1 / 2} e_{n}\right), \quad n=1,2, \ldots
\end{aligned}
$$

imply that

$$
0 \leq \tau\left(e_{n} x^{1 / 2} y x^{1 / 2} e_{n}\right) \leq \tau(|x y|), \quad n=1,2, \ldots .
$$

Since $e_{n} x^{1 / 2} y x^{1 / 2} e_{n} \rightarrow_{n} x^{1 / 2} y x^{1 / 2}$ for the measure topology, Fatou's Lemma implies that $x^{1 / 2} y x^{1 / 2} \in L^{1}(\mathscr{M})$. Since $e_{n} x y \rightarrow_{n} x y$ for the measure topology, the inequalities

$$
\mu\left(e_{n} x y\right) \leq \mu(x y), \quad \mu\left(e_{n} x^{1 / 2} y x^{1 / 2} e_{n}\right) \leq \mu\left(x^{1 / 2} y x^{1 / 2}\right), \quad n=1,2, \ldots,
$$

together with the Dominated Convergence Theorem imply that

$$
\tau\left(x^{1 / 2} y x^{1 / 2}\right)=\lim _{n \rightarrow \infty} \tau\left(e_{n} x^{1 / 2} y x^{1 / 2} e_{n}\right)=\lim _{n \rightarrow \infty} \tau\left(e_{n} x y\right)=\tau(x y) .
$$

Since

$$
x^{1 / 2} y x^{1 / 2}=\left(x^{1 / 2} y^{1 / 2}\right)\left(x^{1 / 2} y^{1 / 2}\right)^{*},
$$

it follows from Lemma 3.1 that

$$
y^{1 / 2} x y^{1 / 2}=\left(x^{1 / 2} y^{1 / 2}\right)^{*}\left(x^{1 / 2} y^{1 / 2}\right)
$$

belongs to $L^{1}(\mathscr{M})$ and that

$$
\tau\left(x^{1 / 2} y x^{1 / 2}\right)=\tau\left(y^{1 / 2} x y^{1 / 2}\right),
$$

and by this the proof is complete.

Lemma 3.5. If $x \geq x_{\alpha} \downarrow_{\alpha} 0$ holds in $\widetilde{\mathscr{M}}$ and if $x \in \widetilde{\mathscr{M}}_{0}$, then $\mu_{t}\left(x_{\alpha}\right) \downarrow_{\alpha} 0$ for all $t>0$.

Proof. If $n \in \mathbb{N}$ and $e=\chi_{(1 / n, n]}(x)$, then $e, e x \in H(\mathscr{M})$. Note that

$$
\mu_{t}(x(1-e)) \leq \frac{1}{n}
$$


on $\left(\tau\left(\chi_{(n, \infty)}(x)\right), \infty\right)$. Now

$$
\begin{aligned}
\mu_{t}^{1 / 2}\left(x_{\alpha}\right) & =\mu_{t}\left(x_{\alpha}^{1 / 2}\right) \leq \mu_{t / 2}\left(x_{\alpha}^{1 / 2} e\right)+\mu_{t / 2}\left(x_{\alpha}^{1 / 2}(1-e)\right) \\
& \leq \mu_{t / 2}^{1 / 2}\left(e x_{\alpha} e\right)+\mu_{t / 2}^{1 / 2}\left((1-e) x_{\alpha}(1-e)\right) \\
& \leq \mu_{t / 2}^{1 / 2}\left(e x_{\alpha} e\right)+\mu_{t / 2}^{1 / 2}(x(1-e)) \\
& \leq \mu_{t / 2}^{1 / 2}\left(e x_{\alpha} e\right)+\frac{1}{\sqrt{n}}, \quad \text { for } t \in\left(2 \tau\left(\chi_{(n, \infty)}(x)\right), \infty\right)
\end{aligned}
$$

Since $x e \geq e x_{\alpha} e \downarrow_{\alpha} 0$ and $x e \in L^{1}(\mathscr{M})$, it follows that

$$
\tau\left(e x_{\alpha} e\right)=\int_{0}^{\infty} \mu_{t}\left(e x_{\alpha} e\right) d t \downarrow_{\alpha} 0
$$

and so $\mu_{t}\left(e x_{\alpha} e\right) \downarrow_{\alpha} 0$ on $(0, \infty)$. Hence $\inf _{\alpha} \mu_{t}\left(x_{\alpha}\right) \leq \frac{1}{n}$ for $t>2 \tau\left(\chi_{(n, \infty)}(x)\right)$ and since $\tau\left(\chi_{(n, \infty)}(x)\right) \downarrow_{n} 0$ as $n \rightarrow \infty$, it follows that $\inf _{\alpha} \mu_{t}\left(x_{\alpha}\right)=0$ on $(0, \infty)$.

The norm on the rearrangement invariant Banach function space $E\left(\mathbb{R}^{+}\right)$is said to be order continuous if and only if whenever $f_{\tau} \downarrow_{\tau} 0$ holds in $E\left(\mathbb{R}^{+}\right)$it follows that $\left\|f_{\tau}\right\|_{E} \downarrow_{\tau} 0$. Order continuity of the norm on the space $E(\mathscr{M})$ is defined similarly.

Proposition 3.6. If $E\left(\mathbb{R}^{+}\right)$is a rearrangement invariant symmetric Banach function space on $\mathbb{R}^{+}$with order continuous norm, then the norm on the space $E(\mathscr{M})$ is order continuous.

Proof. The order continuity of the norm on $E$ implies that $E(\mathscr{M}) \subseteq \widetilde{\mathscr{M}}_{0}$. If now $x_{\alpha} \downarrow_{\alpha} 0$ holds in $E(\mathscr{M})$ then by the preceding lemma it follows that $\mu\left(x_{\alpha}\right) \downarrow_{\alpha} 0$ holds in $E$ and consequently

$$
\left\|x_{\alpha}\right\|_{E(\mathscr{M})}=\left\|\mu\left(x_{\alpha}\right)\right\|_{E} \downarrow_{\alpha} 0 \text {. }
$$

We remark that the preceding Proposition 3.6 has been obtained independently by V. I. Chilin and F. A. Sukochev [CS], in the special case that $\mathscr{M}$ is nonatomic, in which case the converse is also valid.

We now extend the trace to the positive cone of $\widetilde{\mathscr{M}}$.

Definition 3.7. If $0 \leq x \in \widetilde{\mathscr{M}}$, we define $\tau(x)=\sup _{\alpha} \tau\left(x_{\alpha}\right)$, where $\left\{x_{\alpha}\right\}$ is any net in $H(\mathscr{M})$ such that $0 \leq x_{\alpha} \uparrow x$ holds in $\widetilde{\mathscr{M}}$.

The validity of the preceding definition is guaranteed by the following.

Lemma 3.8. If $\left\{x_{\alpha}\right\},\left\{y_{\beta}\right\} \subseteq H(\mathscr{M})$ and if $0 \leq x_{\alpha} \uparrow_{\alpha} x$ and $0 \leq y_{\beta} \uparrow_{\beta} x$ hold in $\widetilde{\mathscr{M}}$ then

$$
\sup _{\alpha} \tau\left(x_{\alpha}\right)=\sup _{\beta} \tau\left(y_{\beta}\right) .
$$

Proof. If $\sup _{\alpha} \tau\left(x_{\alpha}\right)<\infty$ then $x \in L^{1}(\mathscr{M})$ from Proposition 3.3, and so $\sup _{\beta} \tau\left(y_{\beta}\right)<\infty$. Hence in this case

$$
\tau(x)=\sup _{\alpha} \tau\left(x_{\alpha}\right)=\sup _{\beta} \tau\left(y_{\beta}\right) .
$$

The same argument shows that if $\sup _{\alpha} \tau\left(x_{\alpha}\right)=\infty$ then also $\sup _{\beta} \tau\left(y_{\beta}\right)=$ $\infty$.

We remark that the preceding lemma shows that the extension of the trace to $\widetilde{\mathscr{M}_{+}}$given by Definition 3.7 coincides with that given by [FK]. 
Proposition 3.9. (a) The functional $\tau: \widetilde{\mathscr{M}}_{+} \rightarrow[0, \infty]$ is additive, positively homogeneous and unitarily invariant.

(b) If $0 \leq x_{\alpha} \uparrow_{\alpha} x$ holds in $\widetilde{\mathscr{M}}_{+}$then $\tau\left(x_{\alpha}\right) \uparrow_{\alpha} \tau(x)$.

(c) If $x \in \widetilde{\mathscr{M}}$, then

$$
\tau(|x|)=\int_{[0, \infty)} \mu_{t}(x) d t .
$$

(d) If $x \in \widetilde{\mathscr{M}_{0}}$ and if $f$ is any nonnegative Borel function on $\mathbb{R}^{+}$with $f(0)=$ 0 then

$$
\tau(f(|x|))=\int_{[0, \infty)} f\left(\mu_{t}(x)\right) d t .
$$

We remark that parts (a), (b) of the above proposition are straightforward consequences of the definition of $\tau$, while part (c) follows from the remarks preceding Lemma 3.1. The extension of (c) given by (d) has been noted in [FK, Remark 3.3].

We need the following extension of an inequality due to Hardy and Littlewood which in the commutative setting may be found in [Lu, Theorem 8.2] (cf. [DDP1, Proposition 2.3]). The inequality which follows is a consequence of [Fa, Proposition 4.3(ii)] and [FK, Theorem 4.2(iii)], which asserts that if $x, y \in \widetilde{\mathscr{M}}$, then $\mu(x y) \prec \prec \mu(x) \mu(y)$. While this result is of interest in its own right the proof given in [FK] via [Fa] is quite involved. Since the estimate which follows plays such a fundamental role in the subsequent sections, it is desirable for the sake of completeness and clarity to give a somewhat more direct and self-contained proof.

Proposition 3.10. If $x, y \in \widetilde{\mathscr{M}}$ then

$$
\tau(|x y|) \leq \int_{[0, \infty)} \mu_{t}(x) \mu_{t}(y) d t .
$$

Proof. From the equality $\tau(|x y|)=\tau\left(|| x|| y^{*}||\right)$, it may be assumed that $x \geq 0$ and $y \geq 0$. Assume first, in addition, that $0 \leq x, y \in H(\mathscr{M})$. The inequality

$$
\tau(x y) \leq \int_{[0, \infty)} \mu_{t}(x) \mu_{t}(y), d t
$$

is then proved as in [DDP1, Proposition 2.3], and the inequality

$$
\tau(|x y|) \leq \int_{[0, \infty)} \mu_{t}(x) \mu_{t}(y) d t
$$

now follows from (1) and the Cauchy-Schwarz inequality [Di, Lemma I.6.1],

$$
\tau(|x y|)^{2} \leq \tau\left(\left|x^{*} w\right||y|\right) \tau\left(\left|w^{*} x\right|\left|y^{*}\right|\right),
$$

where $x y=w|x y|$ is the polar decomposition of $x y$. If now $0 \leq x \in H(\mathscr{M})$ and $0 \leq y \in L^{1}(\mathscr{M})$ then the stated inequality continues to hold since there exists a sequence $\left\{y_{n}\right\} \subseteq H(\mathscr{M})$ for which

$$
\left\|y-y_{n}\right\|_{1} \rightarrow 0 \text { and } \mu\left(y_{n}\right) \leq \mu(y), \quad n=1,2, \ldots .
$$

We now return to the general case that $0 \leq x, y \in \widetilde{\mathscr{M}}$. It is clear we may assume that

$$
\int_{[0, \infty)} \mu_{t}(x) \mu_{t}(y) d t<\infty,
$$


otherwise there is nothing to prove. It is also clear that it may be assumed that there exists $s>0$ such that $\mu_{s}(x)>0, \mu_{s}(y)>0$, and so from the inequalities

$$
\begin{aligned}
& 0 \leq \mu_{s}(x) \mu(y) \chi_{[0, s)} \leq \mu(x) \mu(y), \\
& 0 \leq \mu_{s}(y) \mu(x) \chi_{[0, s)} \leq \mu(x) \mu(y),
\end{aligned}
$$

it follows that

$$
\int_{[0, s]} \mu_{t}(y) d t<\infty, \quad \text { and } \quad \int_{[0, s]} \mu_{t}(x) d t<\infty,
$$

so that $0 \leq x, y \in G(\mathscr{M})$. Further the condition (2) implies that at least one of $x, y$ belongs to $\widetilde{\mathscr{M}}_{0}$. For definiteness we suppose that $x \in \widetilde{\mathscr{M}}_{0}$. We set $e_{n}=\chi_{(1 / n, n]}(x), n=1,2, \ldots$, and note that

$$
\tau\left(e_{n}\right)<\infty \text { and } x e_{n}=e_{n} x \in H(\mathscr{M}), \quad n=1,2, \ldots
$$

Since $e_{n} x \rightarrow x$ for the measure topology, it follows that $e_{n} x y \rightarrow x y$ for the measure topology. Since $y \in G(\mathscr{M})$ and $\tau\left(e_{n}\right)<\infty$ it follows that $e_{n} y \in$ $L^{1}(\mathscr{M})$ and so from the first part of the proof

$$
\tau\left(\left|e_{n} x y\right|\right)=\tau\left(\left|\left(e_{n} x\right)\left(e_{n} y\right)\right|\right) \leq \int_{[0, \infty)} \mu_{t}(x) \mu_{t}(y) d t, \quad n=1,2, \ldots,
$$

and the desired conclusion now follows from Fatou's Lemma in the form given by Proposition 3.3.

For the case of trace ideals, the inequality of the preceding proposition may be found in [GK, Theorem II 4.2] as special case of Horn's inequality, and in [Ga, Proposition 4].

\section{Contractions for the PAir $\left(L^{1}(\widetilde{\mathscr{M}}), \mathscr{M}\right)$}

The principal aim of this section is to give an exact noncommutative extension of a well-known characterization due to Caldéron [Ca] of spaces which are interpolation spaces for the (commutative) pair $\left(L^{1}, L^{\infty}\right)$. Our approach follows the ideas of Fremlin [Fr] (see also [KPS, Chapter II]) and is based on a separation argument resting on a refinement of the Hardy-Littlewood inequality (Proposition 3.10 above) which is central to the duality theory presented in the sequel.

We suppose that $(\mathscr{N}, \sigma)$ is a semifinite von Neumann algebra with faithful normal semifinite trace $\sigma$ and we denote by $\mathfrak{A}(\mathscr{N}, \mathscr{M})$ the space of bounded linear operators from the Banach couple $\left(L^{1}(\mathscr{N}), \mathscr{N}\right)$ to the Banach couple $\left(L^{1}(\mathscr{M}), \mathscr{M}\right)$. We adhere here to the terminology of [KPS, Chapter I]. With norm defined by setting, for each $T \in \mathfrak{A}(\mathscr{N}, \mathscr{M})$,

$$
\|T\|_{\mathfrak{A}(\mathcal{N}, \mathscr{K})}=\max \left(\left\|T_{1}\right\|,\left\|T_{\infty}\right\|\right),
$$

where $T_{1}, T_{\infty}$ denote the respective restrictions of $T$ to $L^{1}(\mathscr{N}), \mathscr{N}$, it follows that $\mathfrak{A}(\mathscr{N}, \mathscr{M})$ is a Banach space. If $x \in \widetilde{\mathscr{N}}$ and if $y \in \widetilde{\mathscr{M}}$, we will write $y \prec \prec x$ whenever $\mu(y) \prec \prec \mu(x)$. We need the following result, the proof of which follows simply from Proposition 2.5. 
Proposition 4.1. If $T: G(\mathscr{N}) \rightarrow G(\mathscr{M})$ is a linear mapping, then $T \in \mathfrak{A}(\mathscr{N}, \mathscr{M})$ if and only if there exists a positive number $c$ such that $T x \prec \prec c x$, for all $x \in G(\mathcal{N})$, in which case the smallest such positive number $c$ is precisely $\|T\|_{\mathfrak{A}(\mathcal{N}, \mathscr{M})}$.

Let $X$ and $Y$ be Banach spaces and $Y^{*}$ be the dual of $Y$. We denote by $\mathscr{L}(X, Y)$ the linear space of all continuous linear operators from $X$ to $Y$. If $x \in X$ and $y \in Y$, the linear functional $x \otimes y$ is defined on $\mathscr{L}\left(X, Y^{*}\right)$ by setting

$$
\langle T, x \otimes y\rangle=\langle T x, y\rangle, \quad T \in \mathscr{L}\left(X, Y^{*}\right) .
$$

The linear subspace of the algebraic dual $\mathscr{L}\left(X, Y^{*}\right)^{\#}$ generated by functionals of the form $x \otimes y$ is denoted by $X \otimes Y$. It is well known that the unit ball of $\mathscr{L}\left(X, Y^{*}\right)$ is $\sigma\left(\mathscr{L}\left(X, Y^{*}\right), X \otimes Y\right)$ compact. Since the von Neumann algebra $\mathscr{M}$ can be identified with the Banach dual of $L^{1}(\mathscr{M})$ via the canonical pairing [Ta, Theorem V.2.18] it follows immediately that the unit ball of $\mathscr{L}(\mathscr{N}, \mathscr{M})$ is $\sigma\left(\mathscr{L}(\mathscr{N}, \mathscr{M}), \mathscr{N} \otimes L^{1}(\mathscr{M})\right)$ compact.

We denote by $\Sigma(\mathscr{N}, \mathscr{M})$ the set of all linear maps $T \in \mathfrak{A}(\mathscr{N}, \mathscr{M})$ for which $\|T\|_{\mathfrak{A}(\mathcal{N}, \mathscr{M})} \leq 1$.

The following remarks will prove useful in the sequel. It is a consequence of Proposition 2.7(b) that $\mathscr{N}$ is dense in $G(\mathscr{N})$ and so each element of $\mathfrak{A}(\mathscr{N}, \mathscr{M})$ is uniquely determined by its restriction to $\mathscr{N}$. On the other hand, if $S \in$ $\mathscr{L}(\mathscr{N}, \mathscr{M})$ and if there is a constant $c>0$ such that

$$
\|S x\|_{L^{1}(\mathscr{N})} \leq c\|x\|_{L^{1}(\mathcal{N})}, \quad \text { for all } x \in H(\mathscr{N}),
$$

then $S$ has a (unique) extension in $\mathfrak{A}(\mathcal{N}, \mathscr{M})$. In fact, Proposition 2.8 implies that $H(\mathscr{N})$ is dense in $L^{1}(\mathcal{N})$ so that $S$ has a unique extension $S_{1}$ to $L^{1}(\mathscr{N})$ for which $\left\|S_{1}\right\| \leq c$. If $\widetilde{S}: G(\mathscr{N}) \rightarrow G(\mathscr{M})$ is then defined by setting

$$
\widetilde{S}(x+y)=S_{1}(x)+S(y), \quad x \in L^{1}(\mathcal{N}), y \in \mathscr{N},
$$

then it is simple to check that $\widetilde{S}$ is well defined, $\widetilde{S} \in \mathfrak{A}(\mathscr{N}, \mathscr{M})$ and

$$
\|\tilde{S}\|_{\mathfrak{A}(\mathcal{N}, \mathscr{M})} \leq \max \left(\|S\|_{\mathscr{L}(\mathcal{N}, \mathscr{M})}, c\right) .
$$

An immediate consequence of these remarks is that, if $S \in \mathscr{L}(\mathscr{N}, \mathscr{M})$, then $S$ has a unique extension to an element of $\Sigma(\mathscr{N}, \mathscr{M})$ if and only if

$$
|\tau(y S x)| \leq\|x\|_{\mathscr{N}}\|y\|_{L^{1}(\mathscr{K})} \text { and }|\tau(y S x)| \leq\|x\|_{L^{1}(\mathcal{N})}\|y\|_{\mathscr{K}},
$$

for all $x \in H(\mathscr{N}), y \in H(\mathscr{M})$.

Proposition 4.2. $\Sigma(\mathscr{N}, \mathscr{M})$ is $\sigma(\mathfrak{A}(\mathscr{N}, \mathscr{M}), G(\mathscr{N}) \otimes H(\mathscr{M}))$ compact.

Proof. We denote by $\alpha: \mathfrak{A}(\mathscr{N}, \mathscr{M}) \mapsto \mathscr{L}(\mathscr{N}, \mathscr{M})$ the restriction map. It is clear that $\alpha$ is a homeomorphism from $\mathfrak{A}(\mathscr{N}, \mathscr{M})$ onto $\alpha(\mathfrak{A}(\mathscr{N}, \mathscr{M}))$ with respect to the weak topologies induced by $\mathscr{N} \otimes L^{1}(\mathscr{M})$. We show first that $\alpha(\Sigma(\mathscr{N}, \mathscr{M}))$ is a $\sigma\left(\mathscr{L}(\mathscr{N}, \mathscr{M}), \mathscr{N} \otimes L^{1}(\mathscr{M})\right)$ closed subset of the unit ball of $\mathscr{L}(\mathscr{N}, \mathscr{M})$. If

$$
\left\{S_{\beta}\right\} \subseteq \alpha(\Sigma(\mathscr{N}, \mathscr{M})) \text { and } S_{\beta} \rightarrow S
$$

for the topology $\sigma\left(\mathscr{L}(\mathscr{N}, \mathscr{M}), \mathscr{N} \otimes L^{1}(\mathscr{M})\right)$, then it follows immediately that

$$
|\tau(y S x)| \leq\|x\|_{\mathscr{N}}\|y\|_{L^{1}(\mathscr{K})} \text { and }|\tau(y S x)| \leq\|x\|_{L^{1}(\mathcal{N})}\|y\|_{\mathscr{K}},
$$


for all $x \in H(\mathscr{N}), y \in H(\mathscr{M})$. By earlier remarks, it follows that $S \in$ $\alpha(\Sigma(\mathscr{N}, \mathscr{M}))$. It follows therefore that $\Sigma(\mathscr{N}, \mathscr{M})$ is $\sigma\left(\mathfrak{A}(\mathscr{N}, \mathscr{M}), \mathscr{N} \otimes L^{1}(\mathscr{M})\right)$ compact. The proof of the proposition is now completed by observing that the weak topologies induced by $G(\mathscr{N}) \otimes H(\mathscr{M}), \mathscr{N} \otimes L^{1}(\mathscr{K})$ coincide on bounded subsets of $\mathfrak{A}(\mathscr{N}, \mathscr{M})$. This observation is a routine consequence of the fact that $\mathscr{N}, H(\mathscr{M})$ are (norm) dense in $G(\mathscr{N}), L^{1}(\mathscr{M})$ respectively and the details are accordingly omitted.

Corollary 4.3. For each $x \in G(\mathscr{N})$, the subset $\{T x: T \in \Sigma(\mathscr{N}, \mathscr{M})\}$ is $\sigma(G(\mathscr{M}), H(\mathscr{M}))$ compact.

Proof. It suffices to observe that, for each $x \in G(\mathscr{N})$, the evaluation map

$$
T \rightarrow T x, \quad T \in \Sigma(\mathscr{N}, \mathscr{M}),
$$

is $\sigma(\Sigma(\mathscr{N}, \mathscr{M}), G(\mathscr{N}) \otimes H(\mathscr{M}))$ to $\sigma(G(\mathscr{M}), H(\mathscr{M}))$ continuous.

We denote by $\Sigma(\mathscr{N}, \mathscr{M})_{+}$the set of all $T \in \Sigma(\mathscr{N}, \mathscr{M})$ with the property that $T x \geq 0$ in $G(\mathscr{M})$ whenever $x \geq 0$ in $G(\mathscr{N})$.

Lemma 4.4. If $a=\sum_{i=1}^{n} \alpha_{i} e_{i}$ with $e_{1}, \ldots, e_{n}$ mutually orthogonal projections in $H(\mathscr{N})$ and $\alpha_{1} \geq \alpha_{2} \geq \cdots \geq \alpha_{n} \geq 0$, and if $b=\sum_{j=1}^{m} \beta_{j} f_{j}$ with $f_{1}, \ldots, f_{m}$ mutually orthogonal projections in $H(\mathscr{M})$ and $\beta_{1} \geq \beta_{2} \geq \cdots \geq \beta_{m} \geq 0$, then there exists $T \in \Sigma(\mathscr{N}, \mathscr{M})_{+}$such that

Proof. If we write

$$
\int_{[0, \infty)} \mu_{t}(a) \mu_{t}(b) d t=\tau(b T a) .
$$

$$
\begin{aligned}
\xi_{j} & =\sum_{i=1}^{j} \sigma\left(e_{i}\right), & & 1 \leq j \leq n, \\
\eta_{j} & =\sum_{i=1}^{j} \tau\left(f_{i}\right), & & 1 \leq j \leq m,
\end{aligned}
$$

and $\xi_{0}=\eta_{0}=0$, then

$$
\mu(a)=\sum_{i=1}^{n} \alpha_{i} \chi_{\left[\xi_{i-1}, \xi_{i}\right)} \text { and } \mu(b)=\sum_{i=1}^{m} \beta_{i} \chi_{\left[\eta_{i-1}, \eta_{i}\right)} .
$$

We define the linear operator $T: G(\mathscr{N}) \rightarrow G(\mathscr{M})$ by setting

$$
T(x)=\sum_{j=1}^{m}\left(\sum_{i=1}^{n} \frac{\left|\left[\xi_{i-1}, \xi_{i}\right) \cap\left[\eta_{j-1}, \eta_{j}\right)\right|}{\sigma\left(e_{i}\right) \tau\left(f_{j}\right)} \sigma\left(x e_{i}\right)\right) f_{j}, \quad x \in G(\mathscr{N}) .
$$

It is not difficult to show by direct calculation that $T$ has the properties asserted by the lemma and we omit the details.

Theorem 4.5. If $0 \leq x \in G(\mathscr{N})$ and if $0 \leq y \in H(\mathscr{M})$ then

$$
\int_{[0, \infty)} \mu_{t}(x) \mu_{t}(y) d t=\sup \left\{\tau(y T x): T \in \Sigma(\mathscr{N}, \mathscr{M})_{+}\right\} .
$$


Proof. Let $T \in \Sigma(\mathscr{N}, \mathscr{M})_{+}$. Since $T x \prec \prec x$, it follows from the standard Hardy inequality [KPS, II 2.36] that

$$
\int_{[0, \infty)} \mu_{t}(T x) \mu_{t}(y) d t \leq \int_{[0, \infty)} \mu_{t}(x) \mu_{t}(y) d t
$$

and the inequality

$$
\sup \left\{|\tau(y T x)|: T \in \Sigma(\mathscr{N}, \mathscr{M})_{+}\right\} \leq \int_{[0, \infty)} \mu_{t}(x) \mu_{t}(y) d t
$$

now follows from Proposition 3.10. There exist increasing nets $\left\{a_{\alpha}\right\} \subseteq H(\mathscr{N})$, $\left\{b_{\beta}\right\} \subseteq H(\mathscr{M})$ with each $a_{\alpha}$ and $b_{\beta}$ of the form given in Lemma 4.4 for which $0 \leq a_{\alpha} \uparrow_{\alpha} x$ and $0 \leq b_{\beta} \uparrow_{\beta} y$ hold in $\widetilde{\mathscr{N}}, \widetilde{\mathscr{M}}$ respectively, and for each $\alpha, \beta$ there exists $T^{\alpha, \beta} \in \Sigma(\mathscr{N}, \mathscr{M})_{+}$such that

$$
\int_{[0, \infty)} \mu_{t}\left(a_{\alpha}\right) \mu_{t}\left(b_{\beta}\right) d t=\tau\left(b_{\beta} T^{\alpha, \beta} a_{\alpha}\right) .
$$

Since $\mu\left(a_{\alpha}\right) \uparrow_{\alpha} \mu(x)$ and $\mu\left(b_{\beta}\right) \uparrow_{\beta} \mu(y)$ by Proposition 1.7, the assertion of the theorem now follows.

Corollary 4.6. If $x \in G(\mathscr{N})$ and if $y \in H(\mathscr{M})$ then

$$
\begin{aligned}
\int_{[0, \infty)} \mu_{t}(x) \mu_{t}(y) d t & =\sup \{\operatorname{Re} \tau(y T x): T \in \Sigma(\mathscr{N}, \mathscr{M})\} \\
& =\sup \{|\tau(y T x)|: T \in \Sigma(\mathscr{N}, \mathscr{M})\} \\
& =\sup \{\tau(|y T x|): T \in \Sigma(\mathscr{N}, \mathscr{M})\}
\end{aligned}
$$

Proof. For any $T \in \Sigma(\mathscr{N}, \mathscr{M})$, the inequality

$$
\operatorname{Re} \tau(y T x) \leq \int_{[0, \infty)} \mu_{t}(x) \mu_{t}(y) d t
$$

follows as before from the fact that $T x \prec \prec x$. Conversely, it follows from Theorem 4.5 that

$$
\int_{[0, \infty)} \mu_{t}(x) \mu_{t}(y) d t=\sup \left\{\tau(|y| T|x|): T \in \Sigma(\mathscr{N}, \mathscr{M})_{+}\right\}
$$

If $x=u|x|$ and $y=v|y|$ are the polar decompositions of $x, y$ respectively, and if $T \in \Sigma(\mathscr{N}, \mathscr{M})_{+}$, then $\tau(|y| T|x|)=\tau(y \widetilde{T} x)$, where $\widetilde{T} \in \Sigma(\mathscr{N}, \mathscr{M})$ is defined by setting

$$
\widetilde{T} z=T\left(u^{*} z\right) \cdot v^{*}, \quad z \in G(\mathscr{N}),
$$

and the statement of the corollary now follows easily.

For each $x \in \widetilde{\mathscr{N}}$, we define $\Omega_{\mathscr{M}}(x)=\{y \in \widetilde{\mathscr{M}}: y \prec \prec x\}$. We may now state one of the main results of this section.

Theorem 4.7. If $x \in G(\mathscr{N})$, then $\Omega_{\mathscr{M}}(x)=\{T x: T \in \Sigma(\mathscr{N}, \mathscr{M})\}$.

Proof. If $K=\{T x: T \in \Sigma(\mathscr{N}, \mathscr{M})\}$, then it is clear from Proposition 4.1 that $K \subseteq \Omega_{\mathscr{M}}(x)$. Suppose $z \in \Omega_{\mathscr{M}}(x)$ and $z \notin K$. The set $K \subseteq G(\mathscr{M})$ is clearly convex, balanced and is $\sigma(G(\mathscr{M}), H(\mathscr{M}))$ compact by Corollary 4.3 , hence by the Hahn-Banach theorem there exists $y \in H(\mathscr{M})$ such that

$$
\operatorname{Re} \tau(y z)>1 \text { and } \operatorname{Re} \tau(y T x) \leq 1, \quad \text { for all } T \in \Sigma(\mathscr{N}, \mathscr{M}) ;
$$


however, by Corollaries 3.10 and 4.6,

$$
\begin{aligned}
|\operatorname{Re} \tau(y z)| & \leq|\tau(y z)| \leq \tau(|y z|) \leq \int_{[0, \infty)} \mu_{t}(y) \mu_{t}(z) d t \leq \int_{[0, \infty)} \mu_{t}(y) \mu_{t}(x) d t \\
& =\sup \{\operatorname{Re} \tau(y T x): T \in \Sigma(\mathscr{N}, \mathscr{M})\} \leq 1,
\end{aligned}
$$

which is a contradiction.

In the commutative setting, the preceding Theorem 4.7 is due to Caldéron [Ca, Theorems : and 2] for measure algebras which are $\sigma$-finite and to Fremlin [Fr, Theorem 24] for localizable measure algebras. In the noncommutative setting, a restricted form of Theorem 4.7 has been given (essentially) by Ovčinnikov [Ov2] (see also Hiai [Hi] and stated, under present assumptions, in Yeadon [Ye2, Proposition 3.4]; however, the proof given by Yeadon via [Ye2, Lemma 2.3 and Proposition 3.3] fails in general if the trace is not assumed finite.

The following consequence of Theorem 4.7 now follows immediately from Corollary 4.3.

Corollary 4.8. For each $x \in G(\mathscr{N}), \Omega_{\mathscr{M}}(x)$ is convex and $\sigma(G(\mathscr{M}), H(\mathscr{M}))$ compact.

If we note that

$$
\begin{aligned}
& \Sigma(\mathscr{N}, \mathscr{M})_{+}=\{T \in \Sigma(\mathscr{N}, \mathscr{M}): \tau(y T x) \geq 0, \\
&\forall 0 \leq x \in G(\mathscr{N}), \forall 0 \leq y \in H(\mathscr{M})\},
\end{aligned}
$$

then it follows that $\Sigma(\mathscr{N}, \mathscr{M})_{+}$is $\sigma(\mathfrak{A}(\mathscr{N}, \mathscr{M}), G(\mathscr{N}) \otimes H(\mathscr{M}))$ compact and that $\left\{T x: T \in \Sigma(\mathscr{N}, \mathscr{M})_{+}\right\}$is $\sigma(G(\mathscr{M}), H(\mathscr{M}))$ compact for each $0 \leq x \in$ $G(\mathscr{N})$. If we now set

$$
\Omega_{\mathscr{M}}(x)_{+}=\{0 \leq y \in G(\mathscr{M}): y \prec \prec x\},
$$

for all $0 \leq x \in G(\mathscr{N})$, then a variant of the argument of the preceding proposition yields the following result.

Proposition 4.9. If $0 \leq x \in G(\mathscr{N})$, then

(i) $\Omega_{\mathscr{M}}(x)_{+}=\left\{T x: T \in \Sigma(\mathscr{N}, \mathscr{M})_{+}\right\}$,

(ii) $\Omega_{\mathscr{M}}(x)_{+}$is convex and $\sigma(G(\mathscr{M}), H(\mathscr{M}))$ compact.

In the commutative setting, the preceding Corollary 4.8 and Proposition 4.9 are due to Fremlin [Fr, Corollary 7] and are closely related to earlier results of Luxemburg [Lu, Theorem 15.3] and Ryff [Ry, Theorem 2 of $\S 3$ ].

The following decomposition theorem is now an easy consequence of the preceding results. It extends a commutative result due to Lorentz and Shimogaki [LS].

Proposition 4.10. If $y \in G(\mathscr{M})$ and $x_{1}, x_{2} \in G(\mathscr{N})$ are such that $y \prec \prec x_{1}+x_{2}$, then there exist $y_{1}, y_{2} \in G(\mathscr{M})$ such that $y=y_{1}+y_{2}$, and $y_{i} \prec \prec x_{i}, i=1,2$. Moreover, if $y$ is positive, then $y_{1}, y_{2}$ can be taken to be positive.

Proof. If $y \prec \prec x_{1}+x_{2}$ then there exists $T \in \Sigma(\mathscr{N}, \mathscr{M})$ such that

$$
y=T\left(x_{1}+x_{2}\right),
$$

by Theorem 4.7. We simply set $y_{1}=T x_{1}, y_{2}=T x_{2}$. 
Suppose now that $y \geq 0$ and that $y \prec \prec x_{1}+x_{2}$. By [AAP] there exist partial isometries $u_{1}, u_{2} \in \mathscr{N}$ such that $\left|x_{1}+x_{2}\right| \leq u_{1}^{*}\left|x_{1}\right| u_{1}+u_{2}^{*}\left|x_{2}\right| u_{2}$, and so also $0 \leq y \prec \prec u_{1}^{*}\left|x_{1}\right| u_{1}+u_{2}^{*}\left|x_{2}\right| u_{2}$. By Proposition 4.9, there exists $T \in \Sigma(\mathscr{N}, \mathscr{M})_{+}$ such that $y=T\left(u_{1}^{*}\left|x_{1}\right| u_{1}+u_{2}^{*}\left|x_{2}\right| u_{2}\right)$. It clearly suffices to set $y_{i}=T\left(u_{i}^{*}\left|x_{i}\right| u_{i}\right)$, $i=1,2$.

The following sharpening of Proposition 2.5 follows readily from that proposition via Proposition 4.10 preceding.

Corollary 4.11. If $x \in \widetilde{\mathscr{M}}$ and if $\alpha>0$ then

$$
\int_{[0, \alpha]} \mu_{t}(x) d t=\inf \left\{\|y\|_{1}+\alpha\|z\|_{\infty}:|x|=y+z, 0 \leq y \in L^{1}(\mathscr{M}), 0 \leq z \in \mathscr{M}\right\} .
$$

The following is a consequence of Corollary 4.6, Theorems 4.5, 4.7 and Proposition 4.9.

Theorem 4.12. (i) If $x \in G(\mathscr{M})$ and $y \in G(\mathscr{N})$, then

$$
\begin{aligned}
\int_{[0, \infty)} \mu_{t}(x) \mu_{t}(y) d t & =\sup \{|\tau(x z)|: z \in H(\mathscr{M}), z \prec \prec y\} \\
& =\sup \{\tau(|x z|): z \in H(\mathscr{M}), z \prec \prec y\} .
\end{aligned}
$$

(ii) If $0 \leq x \in G(\mathscr{M})$ and $y \in G(\mathscr{N})$, then

$$
\int_{[0, \infty)} \mu_{t}(x) \mu_{t}(y) d t=\sup \{\tau(x z): 0 \leq z \in H(\mathscr{M}), z \prec \prec y\}
$$

Proof. Since

$$
\begin{aligned}
& \sup \{|\tau(x z)|: z \in H(\mathscr{M}), z \prec \prec y\} \\
& \quad=\sup \{|\tau(|x| z)|: z \in H(\mathscr{M}), z \prec \prec y\},
\end{aligned}
$$

we may as well assume that $0 \leq x$. Suppose $\left\{x_{\alpha}\right\} \subseteq H(\mathscr{M})$ satisfies $0 \leq$ $x_{\alpha} \uparrow_{\alpha} x$. If $0 \leq z \in H(\mathscr{M})$ then $z^{1 / 2} x_{\alpha} z^{1 / 2} \uparrow_{\alpha} z^{1 / 2} x z^{1 / 2}$ holds in $L^{1}(\mathscr{M})$ by Propositions 1.3 and 3.2. Consequently $\tau(x z)=\lim _{\alpha} \tau\left(x_{\alpha} z\right)$ holds for ali $z \in H(\mathscr{M})$. By Proposition 1.7 it follows that

$$
\int_{[0, \infty)} \mu_{t}\left(x_{\alpha}\right) \mu_{t}(y) d t \uparrow_{\alpha} \int_{[0, \infty)} \mu_{t}(x) \mu_{t}(y) d t .
$$

The first equality of (i) now follows from Corollary 4.6 and Theorem 4.7. The second equality follows routinely from the first. The assertion of (ii) follows as in (i) via Theorem 4.5 and Proposition 4.9.

\section{KöTHE DUALITY}

The aim of this section is to extend to the noncommutative setting the basic elements of the well-known Köthe duality theory for Banach function spaces as given, for example, in [Za1, Lu]. Related ideas in the trace ideal setting may be found in Garling [Ga] and in [DL]. It is convenient to introduce first some additional terminology.

A Banach space $E \subseteq \widetilde{\mathscr{M}}$ will be called properly symmetric if $E$ is symmetric, rearrangement invariant and intermediate for the Banach couple $\left(L^{1}(\mathscr{M}), \mathscr{M}\right)$ in the sense that $H(\mathscr{M}) \subseteq E \subseteq G(\mathscr{M})$ with continuous embeddings. 
It follows from Proposition 1.4 and Proposition 2.2 that if $E \subseteq \widetilde{\mathscr{M}}$ is a normed rearrangement invariant space then $(E, \leq)$ is a (complex) ordered vector space for which the positive cone is closed and from Proposition 1.1, it follows that $E$ is order complete in the sense that each upwards directed subset with an upper bound in $E$ has at least upper bound in $E$. If $E \subseteq \widetilde{\mathscr{M}}$ is a rearrangement invariant Banach space, it now follows from Ando [An, Theorem 1] that the dual cone generates the Banach dual $E^{*}$; moreover it follows from a well-known argument that each positive linear functional on $E$ is necessarily continuous.

We remark that it is shown in [KPS] that each rearrangement invariant Banach function space $E\left(\mathbb{R}^{+}\right)$is necessarily intermediate for the pair $\left(L^{1}\left(\mathbb{R}^{+}\right)\right.$, $L^{\infty}\left(\mathbb{R}^{+}\right)$) so that if in addition $E\left(\mathbb{R}^{+}\right)$is symmetric then it follows immediately that the operator space $E(\mathscr{M})$ is properly symmetric. It should be noted however that a rearrangement invariant symmetric space need not be intermediate, even in the commutative setting. By way of example, let $\Omega$ be the two point space $\{1,2\}$ equipped with the measure $\nu$ defined on $2^{\Omega}$ by setting $\nu(\{1\})=1, \nu(\{2\})=\frac{1}{2}$, and let $E=\left\{x=\left(x_{1}, x_{2}\right): x_{1}=0\right\}$ and $\|x\|_{E}=\left|x_{2}\right|$, $x \in E$. It is easily checked that $E$ is rearrangement invariant and symmetric. It is clear however that $E$ is not properly symmetric. See also [Ov2, Theorem 3]. On the other hand, it is not difficult to see via Proposition 2.6 that any symmetric rearrangement invariant operator space $E \subseteq \widetilde{\mathscr{M}}$ is necessarily contained in $G(\mathscr{M})$.

Definition 5.1. If $E \subseteq \widetilde{\mathscr{M}}$ is properly symmetric then the Köthe dual $E^{\times}$is defined by setting

$$
E^{\times}=\left\{y \in \widetilde{\mathscr{M}}: x y \in L^{1}(\mathscr{M}) \text { for all } x \in E\right\} .
$$

It is clear that $E^{\times}$is a linear subspace of $\widetilde{\mathscr{M}}$. We gather first some elementary properties.

Proposition 5.2. Let $E \subseteq \widetilde{\mathscr{M}}$ be a properly symmetric Banach space.

(i) If $y \in E^{\times}$and $x \in \mathscr{M}$ then $x y, y x \in E^{\times}$.

(ii) $y \in E^{\times} \Leftrightarrow|y| \in E^{\times} \Leftrightarrow y^{*} \in E^{\times}$.

(iii) $E^{\times}=\left\{y \in \widetilde{\mathscr{M}}: y x \in L^{1}(\mathscr{M})\right.$ for all $\left.x \in E\right\}$.

(iv) If $E \nsubseteq \widetilde{\mathscr{M}}_{0}$ then $E^{\times} \subseteq L^{1}(\mathscr{M})$.

(v) If $x \in E$ and $y \in E^{\times}$then $\tau(x y)=\tau(y x)$.

(vi) If $0 \leq x \in E$ and $0 \leq y \in E^{\times}$, then $x^{1 / 2} y x^{1 / 2}, y^{1 / 2} x y^{1 / 2} \in L^{1}(\mathscr{M})$ and

$$
\tau(x y)=\tau\left(x^{1 / 2} y x^{1 / 2}\right)=\tau\left(y^{1 / 2} x y^{1 / 2}\right) \geq 0 .
$$

(vii) If $y \in E^{\times}$then the linear functional $x \rightarrow \tau(x y), x \in E$, is continuous.

(viii) $L^{1}(\mathscr{M})^{\times}=\mathscr{M}$ and $\mathscr{M}^{\times}=L^{1}(\mathscr{M})$.

(ix) $G(\mathscr{M})^{\times}=H(\mathscr{M})$ and $H(\mathscr{M})^{\times}=G(\mathscr{M})$.

(x) $H(\mathscr{M}) \subseteq E^{\times} \subseteq G(\mathscr{M})$.

Proof. Statements (i)-(iii) follow by routine arguments. If $E \nsubseteq \widetilde{\mathscr{M}_{0}}$ then $1 \in E$ and this implies that $E^{\times} \subseteq L^{1}(\mathscr{M})$, and so (iv) follows. Statements (v) and (vi) are consequences of Proposition 3.4, (vii) follows from (vi) and ( $x$ ) is a consequence of (ix). To prove (viii), observe first that if $0 \leq y \in L^{1}(\mathscr{M})^{\times}$, then the linear functional $\phi$ defined via

$$
\phi(x)=\tau(x y), \quad x \in L^{1}(\mathscr{M}),
$$


is continuous by (vii). If $y \notin \mathscr{M}$ then for every $\lambda>0$, there exists $e \in \mathscr{M}^{p}$ such that $y e=e y e \geq \lambda e$ and so

$$
\phi(e)=\tau(y e) \geq \lambda \tau(e)=\lambda\|e\|_{1},
$$

and this contradicts the continuity of $\phi$. Consequently $L^{1}(\mathscr{M})^{\times} \subseteq \mathscr{M}$ and since the reverse inclusion is trivial, it follows that $L^{1}(\mathscr{M})^{\times}=\mathscr{M}$. If now $y \in \mathscr{M}^{\times}$, then $y \in L^{1}(\mathscr{M})$, since $1 \in \mathscr{M}$. The inclusion $L^{1}(\mathscr{M}) \subseteq \mathscr{M}^{\times}$is trivial and it follows that $\mathscr{M}^{\times}=L^{1}(\mathscr{M})$. The first assertion of (ix) follows directly from (viii) while the second equality of (ix) is a simple consequence of the characterization given in Proposition 2.6.

If the Banach space $E \subseteq \widetilde{\mathscr{M}}$ is properly symmetric and if $x \in \widetilde{\mathscr{M}}$, we define

$$
\|x\|_{E^{\times}}=\sup \left\{\tau(|x y|): y \in E,\|y\|_{E} \leq 1\right\} .
$$

Proposition 5.3. Let $E \subseteq \widetilde{\mathscr{M}}$ be a properly symmetric Banach space.

(i) If $x \in \widetilde{\mathscr{M}}$, then

$$
\begin{aligned}
\|x\|_{E^{\times}} & =\sup \left\{\tau(|x y|): y \in H(\mathscr{M}),\|y\|_{E} \leq 1\right\} \\
& =\sup \left\{\int_{[0, \infty)} \mu_{t}(x) \mu_{t}(y) d t: y \in H(\mathscr{M}),\|y\|_{E} \leq 1\right\} \\
& =\sup \left\{\int_{[0, \infty)} \mu_{t}(x) \mu_{t}(y) d t: y \in E,\|y\|_{E} \leq 1\right\} .
\end{aligned}
$$

(ii) If $x \in \widetilde{\mathscr{M}}$, then $x \in E^{\times}$if and only if $\|x\|_{E^{\times}}<\infty$, in which case,

$$
\begin{aligned}
\|x\|_{E^{\times}} & =\sup \left\{|\tau(x y)|: y \in E,\|y\|_{E} \leq 1\right\} \\
& =\sup \left\{|\tau(x y)|: y \in H(\mathscr{M}),\|y\|_{E} \leq 1\right\} .
\end{aligned}
$$

Proof. (i) Let $x \in \widetilde{\mathscr{M}}$. From Proposition 3.10 we may assume that

$$
\sup \left\{\tau(|x y|): y \in H(\mathscr{M}),\|y\|_{E} \leq 1\right\}<\infty ;
$$

and so necessarily $x \in G(\mathscr{M})$ and consequently it follows from Propositions $1.7,1.8$ and Theorem 4.12 that

$$
\begin{aligned}
\sup & \left\{\int_{[0, \infty)} \mu_{t}(x) \mu_{t}(y) d t: y \in E,\|y\|_{E} \leq 1\right\} \\
& =\sup \left\{\int_{[0, \infty)} \mu_{t}(x) \mu_{t}(y) d t: y \in H(\mathscr{M}),\|y\|_{E} \leq 1\right\} \\
& =\sup \left\{\tau(|x z|): \exists y \in H(\mathscr{M}),\|y\|_{E} \leq 1 \text { and } z \prec \prec y\right\} \\
& =\sup \left\{\tau(|x y|): y \in H(\mathscr{M}),\|y\|_{E} \leq 1\right\} .
\end{aligned}
$$

(ii) If $x \in \widetilde{\mathscr{M}}$ and $\|x\|_{E^{\times}}<\infty$, then it is clear that $x \in E^{\times}$and that

$$
\sup \left\{|\tau(x y)|: y \in E,\|y\|_{E} \leq 1\right\} \leq\|x\|_{E^{\times}} .
$$

On the other hand, if $x \in E^{\times}$and if $y \in E$, and $\|y\|_{E} \leq 1$, let $x y=u|x y|$ be the polar decomposition of $x y$; it follows that

$$
\tau\left(x y u^{*}\right)=\tau(|x y|) \text { and }\left\|y u^{*}\right\|_{E} \leq 1,
$$


and from this we obtain

$$
\|x\|_{E^{\times}} \leq \sup \left\{|\tau(x y)|: y \in E,\|y\|_{E} \leq 1\right\} .
$$

This latter supremum is finite via Proposition 5.2(vii). The final equality in the assertion of (ii) is a consequence of the normality of the trace.

Proposition 5.4. Let $E \subseteq \widetilde{\mathscr{M}}$ be a properly symmetric Banach space.

(i) If $x \in \widetilde{\mathscr{M}}, y \in E^{\times}$, and $x \prec \prec y$ then $x \in E^{\times}$and $\|x\|_{E^{\times}} \leq\|y\|_{E^{\times}}$.

(ii) $0 \leq x_{\alpha} \uparrow_{\alpha} x \in \widetilde{\mathscr{M}}$ implies $\left\|x_{\alpha}\right\|_{E^{\times}} \uparrow_{\alpha}\|x\|_{E^{\times}}$.

(iii) $\left(\bar{E}^{\times},\|\cdot\|_{E^{\times}}\right)$is a properly symmetric Banach space.

Proof. Part (i) follows from Proposition 5.3 and [KPS, II 2.18]. If $0 \leq x_{\alpha} \uparrow_{\alpha}$ $x \in \widetilde{\mathscr{M}}$, then $\mu\left(x_{\alpha}\right) \uparrow_{\alpha} \mu(x)$, and (ii) now follows from Proposition 5.3. Finally, (iii) follows from (ii) and Corollary 2.4 .

If $E \subseteq \widetilde{\mathscr{M}}$ is a properly symmetric Banach space then $E$ will be called muximal if and only if the natural embedding of $E$ into the Köthe bidual $E^{\times \times}$is a surjective isometry. We may now state the following representationtype result, due in the commutative setting to Luxemburg [Lu], to which we refer for the essential details.

Theorem 5.5. If $E \subseteq \widetilde{\mathscr{M}}$ is a properly symmetric Banach space then there exists a maximal properly symmetric Banach function space $F\left(\mathbb{R}^{+}\right)$such that $E^{\times}=$ $F(\mathscr{M})$.

Proof. It is not difficult to see that $F\left(\mathbb{R}^{+}\right)$may be taken to be the linear subspace of $L^{0}\left(\mathbb{R}^{+}\right)$consisting of all $f \in L^{0}\left(\mathbb{R}^{+}\right)$for which

$$
\|f\|_{F\left(\mathbf{R}^{+}\right)}:=\sup \left\{\int_{[0, \infty)} \mu_{t}(f) \mu_{t}(y) d t: y \in E,\|y\|_{E} \leq 1\right\}<\infty .
$$

The function norm $\|\cdot\|_{F\left(\mathbb{R}^{+}\right)}$has the (so-called) Fatou property:

$$
0 \leq f_{\alpha} \uparrow_{\alpha} f \in L^{0}\left(\mathbb{R}^{+}\right) \quad \text { implies } \quad\left\|f_{\alpha}\right\|_{F\left(\mathbb{R}^{+}\right)} \uparrow_{\alpha}\|f\|_{F\left(\mathbf{R}^{+}\right)}
$$

and it is well known that this property implies that $F\left(\mathbb{R}^{+}\right)$is maximal. For example, see [KPS] or [Za1].

For ease of notation, we write $E^{\times}\left(\mathbb{R}^{+}\right)$for the Köthe dual of the properly symmetric Banach function space $E\left(\mathbb{R}^{+}\right)$.

Theorem 5.6. If $E\left(\mathbb{R}^{+}\right)$is a properly symmetric Banach function space on $\mathbb{R}^{+}$, then the equality $E^{\times}(\mathscr{M})=E(\mathscr{M})^{\times}$holds in the sense of Banach spaces.

Proof. The inclusion $E^{\times}(\mathscr{M}) \subseteq E(\mathscr{M})^{\times}$together with the norm inequality

$$
\|x\|_{E^{\times}(\mathscr{M})} \geq\|x\|_{E(\mathscr{M})^{\times}}, \quad x \in E^{\times}(\mathscr{M}),
$$

follows directly from the relevant definitions and Proposition 5.3. If $x \in$ $E(\mathscr{M})^{\times}$, it follows from Theorem 4.12 that

$$
\begin{aligned}
\sup & \left\{\int_{[0, \infty)} \mu_{t}(x) \mu_{t}(f) d t: f \in H\left(\mathbb{R}^{+}\right),\|f\|_{E\left(\mathbf{R}^{+}\right)} \leq 1\right\} \\
& =\sup \left\{|\tau(x y)|: y \in H(\mathscr{M}), \quad \exists f \in H\left(\mathbb{R}^{+}\right),\|f\|_{E\left(\mathbf{R}^{+}\right)} \leq 1, y \prec \prec f\right\} .
\end{aligned}
$$


Since $E\left(\mathbb{R}^{+}\right)$is symmetric, this latter supremum is at most

$$
\sup \left\{|\tau(x y)|: y \in H(\mathscr{M}),\|y\|_{E(\mathscr{M})} \leq 1\right\}
$$

which is finite, as follows from Proposition 5.2(vii). It now follows from Proposition 5.3 that $x \in E^{\times}(\mathscr{M})$ and that $\|x\|_{E^{\times}(\mathscr{K})} \leq\|x\|_{E(\mathscr{K})^{\times}}$.

The preceding Proposition 5.6 permits ready identification of the Köthe dual of a (noncommutative) properly symmetric space, which is constructed from a given properly symmetric Banach function space on $\mathbb{R}^{+}$. For example, we obtain immediately the Banach space equalities

$$
\left(L^{1}(\mathscr{M})+\mathscr{M}\right)^{\times}=L^{1}(\mathscr{M}) \cap \mathscr{M}, \quad\left(L^{1}(\mathscr{M}) \cap \mathscr{M}\right)^{\times}=L^{1}(\mathscr{M})+\mathscr{M},
$$

which follow via Proposition 5.6 from their corresponding commutative specializations, which are standard facts in interpolation theory given, for example, in [KPS, II, 3.1].

Before proceeding, we mention the following further consequence of Propositions 5.5 and 5.6.

Corollary 5.7. If $E \subseteq \widetilde{\mathscr{M}}$ is a properly symmetric Banach space then $E^{\times}$is maximal.

If $E \subseteq \widetilde{\mathscr{M}}$ is a properly symmetric operator space, it is clear that the mapping $\Phi: E^{\times} \rightarrow E^{*}$ defined by setting $\Phi(y)(x)=\tau(x y), x \in E$, for each $y \in E^{\times}$ is an injective isometry onto a linear subspace of the Banach dual $E^{*}$. It is moreover not difficult to see that if $y \in E^{\times}$then $\Phi(y)(x) \geq 0$ for all $0 \leq x \in E$ if and only if $y \geq 0$. We turn now to the question of characterizing those elements of the Banach dual $E^{*}$ which are given by elements of the Köthe dual via the above pairing. To this end we make the following definition.

Definition 5.8. If $E \subseteq \widetilde{\mathscr{M}}$ is a properly symmetric Banach space and $\psi \in E^{*}$, then $\psi$ is called

(i) normal if and only if

$$
x_{\alpha} \downarrow_{\alpha} 0 \subseteq E \text { implies } \psi\left(x_{\alpha}\right) \rightarrow 0 .
$$

(ii) completely additive if and only if, whenever $x \in E$,

$$
e_{\alpha} \downarrow_{\alpha} 0 \subseteq \mathscr{M}^{p} \text { implies } \psi\left(x e_{\alpha}\right) \rightarrow 0 \text { and } \psi\left(e_{\alpha} x\right) \rightarrow 0 .
$$

The linear space of normal linear functionals on $E$ will be denoted by $E_{n}^{*}$. It is well known in the case $E=\mathscr{M}$ that if $\psi \in E^{*}$ then $\psi$ is normal (respectively completely additive) if and only if $\psi$ is continuous for the ultra-weak topology on $\mathscr{M}$ [SZ, Theorem 5.11], and it then follows from [Di, I 6.10] that there exists a unique element $a \in L^{1}(\mathscr{M})$ such that $\psi=\Phi(a)$. We write $H(\mathscr{M})^{p}$ for $\mathscr{M}^{p} \cap H(\mathscr{M})$.

Lemma 5.9. Let $E \subseteq \widetilde{\mathscr{M}}$ be a properly symmetric Banach space and let $F\left(\mathbb{R}^{+}\right)$ be a maximal properly symmetric Banach function space on $\mathbb{R}^{+}$for which $F(\mathscr{M})$ $=E^{\times}$. If $\psi \in E^{*}$ and if $\psi$ is either normal or completely additive then there exists $f \in F\left(\mathbb{R}^{+}\right)$such that

$$
|\psi(x)| \leq \int_{[0, \infty)} \mu_{t}(x) f(t) d t
$$

for all $x \in E$. 
Proof. We show first that there exists $0 \leq f \in F\left(\mathbb{R}^{+}\right)$, such that

$$
|\psi(x)| \leq \int_{[0, \infty)} \mu_{t}(x) f(t) d t
$$

for all $0 \leq x \in H(\mathscr{M})$. To this end, if $e \in H(\mathscr{M})^{p}$, observe that the functional $z \rightarrow \psi(e z e), z \in \mathscr{M}$, is either normal or completely additive on $\mathscr{M}$, and consequently, there exists a unique element $a_{e} \in L^{1}(\mathscr{M})$ such that

$$
\psi(e z e)=\tau\left(z a_{e}\right)=\tau\left(a_{e} z\right), \quad \text { for all } z \in \mathscr{M} .
$$

If now $e, e^{\prime} \in H(\mathscr{M})^{p}$ and if $e^{\prime} \leq e$, then a simple uniqueness argument shows that $a_{e^{\prime}}=e^{\prime} a_{e} e^{\prime}$, and from this it follows further that $\mu\left(a_{e^{\prime}}\right) \leq \mu\left(a_{e}\right)$. If now $z \in H(\mathscr{M})$ and $\|z\|_{E} \leq 1$, observe that Theorem 4.12(i) implies

$$
\int_{[0, \infty)} \mu_{t}\left(a_{e}\right) \mu_{t}(z) d t \leq \sup \left\{\left|\tau\left(y a_{e}\right)\right|: y \in H(\mathscr{M}),\|y\|_{E} \leq 1\right\} \leq\|\psi\| .
$$

It follows that the system $\left\{\mu\left(a_{e}\right)\right\}, e \in H(\mathscr{M})^{p}$, is upwards directed and norm bounded in $F\left(\mathbb{R}^{+}\right)$. Since $F\left(\mathbb{R}^{+}\right)$is maximal, it follows that there exists $0 \leq$ $f \in F\left(\mathbb{R}^{+}\right)$such that $0 \leq \mu\left(a_{e}\right) \uparrow f$ holds in $F\left(\mathbb{R}^{+}\right)$. If $0 \leq x \in H(\mathscr{M})$, there exists $e_{n} \uparrow_{n} \subseteq H(\mathscr{M})^{p}$ such that $e_{n} x=x e_{n} \uparrow_{n} x$. It then follows that

$$
\begin{aligned}
\left|\psi\left(e_{n} x e_{n}\right)\right| & =\left|\tau\left(x a_{e_{n}}\right)\right| \leq \int_{[0, \infty)} \mu_{t}(x) \mu_{t}\left(a_{e_{n}}\right) d t \\
& \leq \int_{[0, \infty)} \mu_{t}(x) f(t) d t, \quad n=1,2, \ldots
\end{aligned}
$$

It follows from either normality or complete additivity that the inequality

$$
|\psi(x)| \leq \int_{[0, \infty)} \mu_{t}(x) f(t) d t
$$

holds for all $0 \leq x \in H(\mathscr{M})$. If $\psi$ is normal, then the above inequality continues to hold for all $0 \leq x \in E$, as follows from Propositions 1.7, 1.8. On the other hand, assume that $\psi$ is completely additive. If $0 \leq x \in \mathscr{M} \cap E$ and if $\left\{e_{\alpha}\right\} \subseteq H(\mathscr{M})^{p}$ is any system for which $e_{\alpha} \uparrow_{\alpha} 1$ then $\left\{x e_{\alpha}\right\} \subseteq H(\mathscr{M})$ and complete additivity of $\psi$ now implies that the stated inequality continues to hold for all $0 \leq x \in \mathscr{M} \cap E$. Finally, if $0 \leq x \in E$, then there exists a sequence $\left\{e_{n}\right\}$ of spectral projections of $x$ such that $0 \leq e_{n} \uparrow_{n} 1$ and such that $x e_{n} \in \mathscr{M}, n=1,2, \ldots$, and the inequality

$$
|\psi(x)| \leq \int_{[0, \infty)} \mu_{t}(x) f(t) d t
$$

again follows from the complete additivity of $\psi$. This clearly suffices to prove the assertion of the lemma.

Lemma 5.10. If $\psi \in E_{n}^{*}$ and if $e \in H(\mathscr{M})^{p}$, then the functional $\psi_{e}$ defined by setting $\psi_{e}(x)=\psi(e x), x \in \mathscr{M}$, is a normal linear functional on $\mathscr{M}$.

Proof. It is not difficult to see that $\psi_{e} \in \mathscr{M}^{*}$. If $x_{0} \geq x_{\alpha} \downarrow_{\alpha} 0$ holds in $\mathscr{M}$, then $e x_{\alpha} e \downarrow_{\alpha} 0$ holds in $L^{1}(\mathscr{M})$, and consequently $\mu\left(e x_{\alpha} e\right) \downarrow_{\alpha} 0$ holds in $L^{1}\left(\mathbb{R}^{+}\right)$. Suppose that $0 \leq f \in F\left(\mathbb{R}^{+}\right)$is as given by the preceding Lemma 5.9. Since

$$
\mu\left(e x_{\alpha}\right)=\mu\left(x_{\alpha} e\right)=\mu^{1 / 2}\left(\left|x_{\alpha} e\right|^{2}\right)=\mu^{1 / 2}\left(e x_{\alpha}^{2} e\right) \leq\left\|x_{0}\right\|^{1 / 2} \mu^{1 / 2}\left(e x_{\alpha} e\right)
$$


and since $\mu^{1 / 2}\left(e x_{\alpha} e\right) \in H\left(\mathbb{R}^{+}\right)$, it follows from the inequalities

$$
\left|\psi_{e}\left(x_{\alpha}\right)\right| \leq \int_{[0, \infty)} \mu_{t}\left(e x_{\alpha}\right) f(t) d t \leq\left\|x_{0}\right\|^{1 / 2} \int_{[0, \infty)} \mu_{t}^{1 / 2}\left(e x_{\alpha} e\right) f(t) d t,
$$

that $\psi_{e}\left(x_{\alpha}\right) \rightarrow 0$, and the proof is complete.

Theorem 5.11. If $E \subseteq \widetilde{\mathscr{M}}$ is a properly symmetric Banach space and if $\psi \in E^{*}$, then the following statements are equivalent.

(i) $\psi$ is completely additive.

(ii) There exists $a \in E^{\times}$such that $\psi(x)=\tau(a x)$, for all $x \in E$, in which case $\|\psi\|=\|a\|_{E^{\times}}$.

(iii) $\psi$ is normal.

Proof. (ii) $\Rightarrow$ (iii). It clearly suffices to assume in addition that $a \geq 0$. If $0 \leq$ $x_{\alpha} \downarrow_{\alpha} 0$ holds in $E$ then $a^{1 / 2} x_{\alpha} a^{1 / 2} \downarrow_{\alpha} 0$ holds in $L^{1}(\mathscr{M})$, and consequently

$$
\tau\left(a x_{\alpha}\right)=\tau\left(a^{1 / 2} x_{\alpha} a^{1 / 2}\right) \downarrow_{\alpha} 0
$$

The proof of the implication (ii) $\Rightarrow$ (i) is similar to the proof of the implication (ii) $\Rightarrow$ (iii) .

(iii) $\Rightarrow$ (ii) . It may be assumed without loss of generality that the functional $\psi \in E_{n}^{*}$ is selfadjoint, that is

$$
\psi\left(x^{*}\right)=\overline{\psi(x)}, \quad x \in E .
$$

If now $e \in H(\mathscr{M})^{p}$, define $\psi_{e}$ by setting

$$
\psi_{e}(x)=\psi(e x), \quad x \in \mathscr{M} .
$$

From the preceding Lemma 5.10 it follows that $\psi_{e} \in \mathscr{M}_{n}^{*}$, and consequently there exists a unique $a_{e} \in L^{1}(\mathscr{M})$ such that

$$
\psi_{e}(x)=\psi(e x)=\tau\left(a_{e} x\right), \quad \text { for all } x \in \mathscr{M} .
$$

As in the proof of [Y1, Theorem 4.3], a simple argument shows that

(a) $a_{e^{\prime}}=a_{e} e^{\prime}$ whenever $e, e^{\prime} \in H(\mathscr{M})^{p}$ and $e^{\prime} \leq e$.

(b) $e a_{e}$ is selfadjoint whenever $e \in H(\mathscr{M})^{p}$.

The operator $a_{0}$ is defined by setting

$$
D\left(a_{0}\right)=\bigcup\left\{D\left(a_{e}\right) \cap e(H): e \in H(\mathscr{M})^{p}\right\},
$$

and

$$
a_{0} \xi=a_{e} \xi, \quad \text { for } \xi \in D\left(a_{e}\right) \cap e(H) .
$$

The argument of the first part of the proof of [Ye2, Theorem 4.2] shows that

(1) $a_{0}$ is well defined, densely defined, linear and symmetric;

(2) if $a$ is defined to be the closure of $a_{0}$, then $a$ is symmetric, affiliated with $\mathscr{M}$ and $a_{e}=a e$, for $e \in H(\mathscr{M})^{p}$.

If $a=u|a|$ is the polar decomposition of $a$, then $|a|$ is affiliated with and the spectral resolution of $|a|$ is contained in $\mathscr{M}$. To show that $a \in \widetilde{\mathscr{M}}$, observe that if $e \in H(\mathscr{M})^{p}$, then

$$
\tau(e|a| e)=\tau\left(u^{*} a e\right)=\psi\left(e u^{*}\right) \leq\|\psi\|\|e\|_{E} .
$$

There exists a constant $c>0$ such that

$$
\|x\|_{E} \leq c\|x\|_{H(\mathscr{M})}, \quad \text { for all } x \in H(\mathscr{M}),
$$


and consequently

$$
\tau(e|a| e) \leq c\|\psi\| \max (\tau(e), 1), \quad \text { for all } e \in H(\mathscr{M})^{p} .
$$

If $\lambda>c\|\psi\|$ and $e \in H(\mathscr{K})^{p}$ satisfies $0 \leq e \leq \chi_{(\lambda, \infty)}(|a|)$, then

$$
\lambda \tau(e) \leq \tau(e|a| e) \leq c\|\psi\| \max (\tau(e), 1) .
$$

It follows that $\tau(e) \leq 1$, so that also $\tau\left(\chi_{(\lambda, \infty)}(|a|)\right) \leq 1$ for all $\lambda>c\|\psi\|$. Hence $a \in \widetilde{\mathscr{M}}$, and since $a$ is symmetric, it follows as well that $a$ is selfadjoint, by [Te, Corollary I 15]. Suppose now that $0 \leq x \in H(\mathscr{M})$. There exists $0 \leq e_{n} \uparrow_{n} \subseteq H(\mathscr{M})^{p}$ such that $e_{n} x=x e_{n} \uparrow_{n} x$ and such that $a e_{n} x \rightarrow a x$ for the measure topology. Writing

$$
\left|a e_{n} x-a e_{m} x\right|=w_{n, m}\left(a e_{n} x-a e_{m} x\right),
$$

with $w_{n, m} \in \mathscr{M}$ a partial isometry for $n, m=1,2, \ldots$, observe that

$$
\begin{aligned}
\tau\left(\left|a e_{n} x-a e_{m} x\right|\right) & =\tau\left(\left(a e_{n} x-a e_{m} x\right) w_{m, n}\right)=\psi\left(\left(e_{n} x-e_{m} x\right) w_{m, n}\right) \\
& \leq \int_{[0, \infty)} \mu_{t}\left(e_{n} x-e_{m} x\right) f(t) d t,
\end{aligned}
$$

where the function $f$ is as given by Lemma 5.9 above. It follows that

$$
\left\|a e_{n} x-a e_{m} x\right\|_{1} \rightarrow 0 \text { as } n, m \rightarrow \infty,
$$

and since $a e_{n} x \rightarrow a x$ for the measure topology, it follows that $a x \in L^{1}(\mathscr{M})$ and that

$$
\psi\left(e_{n} x\right)=\tau\left(a e_{n} x\right) \rightarrow \tau(a x) .
$$

On the other hand, by normality of $\psi$, it follows that $\psi\left(e_{n} x\right) \rightarrow \psi(x)$ as $n \rightarrow \infty$ and so the equality $\psi(x)=\tau(a x)$ holds for all $0 \leq x \in H(\mathscr{M})$. The preceding argument shows as well that $a \in H(\mathscr{M})^{\times}=G(\mathscr{M})$. If now $x \in H(\mathscr{M})$, then it follows from Theorem 4.12 that

$$
\int_{[0, \infty)} \mu_{t}(a) \mu_{t}(x) d t=\sup \{|\tau(a y)|: y \in H(\mathscr{M}), \quad y \prec \prec x\} \leq\|\psi\|\|x\|_{E} .
$$

Consequently,

$$
\|a\|_{E^{\times}}=\sup \left\{\int_{[0, \infty)} \mu_{t}(a) \mu_{t}(x) d t: x \in H(\mathscr{M}),\|x\|_{E} \leq 1\right\}<\infty,
$$

and this implies that $a \in E^{\times}$and that $\|a\|_{E^{\times}}=\|\psi\|$, by Proposition 5.3.

Finally, the proof of the implication (i) $\Rightarrow$ (ii) is almost identical to the proof of the implication (iii) $\Rightarrow$ (ii), with the complete additivity of $\psi$ making an appeal to Lemma 5.10 unnecessary.

The preceding theorem yields an extension of Proposition 3.2, and to formulate this extension, it is convenient to introduce the following terminology. If $E \subseteq \widetilde{\mathscr{M}}$ is properly symmetric, then $E$ is said to have the Beppo-Levi property if and only if $0 \leq x_{\alpha} \uparrow_{\alpha} \subseteq E$, sup $\left\|x_{\alpha}\right\|_{E}<\infty$ implies $\sup x_{\alpha}$ exists in $E$. 
Corollary 5.12. Let $E\left(\mathbb{R}^{+}\right)$be a properly symmetric Banach function space on $\mathbb{R}^{+}$. If $E\left(\mathbb{R}^{+}\right)$has the Beppo-Levi property, then so does $E(\mathscr{M})$.

Proof. Assume that $0 \leq x_{\alpha} \uparrow_{\alpha} \subseteq E(\mathscr{M})$ and that $\sup _{\alpha}\left\|x_{\alpha}\right\|_{E}<\infty$. For $0 \leq$ $y \in E(\mathscr{M})^{\times}$, define $\psi(y)=\sup _{\alpha} \tau\left(x_{\alpha} y\right)$. The functional $\psi$ is additive and positively homogeneous on the positive cone of $E(\mathscr{M})^{\times}$, and so extends by linearity to a positive linear functional on $E(\mathscr{M})^{\times}$, which we continue to denote by $\psi$. A routine calculation shows that $0 \leq \psi \in\left(E(\mathscr{M})^{\times}\right)_{n}^{*}$, and that $\|\psi\| \leq$ $\sup _{\alpha}\left\|x_{\alpha}\right\|_{E^{\times \times(\mathscr{C})}} \leq \sup _{\alpha}\left\|x_{\alpha}\right\|_{E}$. Consequently, by Theorem 5.11 and the remark following Corollary 5.7, there exists $0 \leq x \in E^{\times \times}(\mathscr{M})$ such that the equality $x=\sup x_{\alpha}$ holds in $E^{\times \times}(\mathscr{M})$. From Proposition 1.7, it follows that $\mu\left(x_{\alpha}\right) \uparrow_{\alpha}$ $\mu(x)$ and since $E\left(\mathbb{R}^{+}\right)$has the Beppo-Levi property, it follows that $\mu(x) \in$ $E\left(\mathbb{R}^{+}\right)$, and this suffices to complete the proof.

Special cases of the preceding Corollary 5.12 have been obtained by V. I. Chilin and F. Sukochev (personal communication; see also [GS] and the references contained therein).

Before making some remarks, it is convenient to introduce some further terminology. The norm on the properly symmetric Banach space $E \subseteq \widetilde{\mathscr{M}}$ will be called order continuous if and only if whenever $\left\{x_{\alpha}\right\} \subseteq E$ and $0 \leq x_{\alpha} \downarrow_{\alpha} 0$ holds in $E$, it follows that $\left\|x_{\alpha}\right\|_{E} \downarrow_{\alpha} 0$. It follows from a standard argument (see, for example, [ $\mathrm{Za} 2$, Lemma 102.5]) that the norm on $E$ is order continucus if and only if $E^{*}=E_{n}^{*}=E^{\times}$. Suppose now that $E\left(\mathbb{R}^{+}\right)$is a rearrangement invariant symmetric Banach function space on $\mathbb{R}^{+}$. If the norm on $E\left(\mathbb{R}^{+}\right)$is order continuous, then it follows from Proposition 3.6 that the norm on $E(\mathscr{M})$ is also order continuous and so Theorem 5.11 combined with Theorem 5.6 shows that the Banach dual $E(\mathscr{M})^{*}$ may be identified with the space $E^{\times}(\mathscr{M})$. Since the norm on each of the spaces $L^{p}\left(\mathbb{R}^{+}\right), 1 \leq p<\infty$ is order continuous the usual identification

$$
L^{p}(\mathscr{M})^{*}=L^{q}(\mathscr{M}), \quad 1 \leq p<\infty, \frac{1}{p}+\frac{1}{q}=1,
$$

follows immediately. To make some further remarks, we suppose that $\psi$ is an increasing concave function on $\mathbb{R}^{+}$with $\psi(0+)=0$ and $\psi(\infty)=\infty$. Following [KPS, Chapter II.5], we let $\Lambda_{\psi}\left(\mathbb{R}^{+}\right), M_{\psi}\left(\mathbb{R}^{+}\right)$be the usual Lorentz and Marcinkiewicz spaces with norms defined by setting

$$
\begin{gathered}
\|f\|_{\Lambda_{\psi}\left(\mathbb{R}^{+}\right)}=\int_{[0, \infty)} \mu_{t}(f) \psi^{\prime}(t) d t, \quad f \in \Lambda_{\psi}\left(\mathbb{R}^{+}\right), \\
\|f\|_{M_{\psi}\left(\mathbf{R}^{+}\right)}=\sup _{\alpha>0} \frac{1}{\psi(\alpha)} \int_{0}^{\alpha} \mu_{t}(f) d t .
\end{gathered}
$$

It follows from [KPS, Corollary 1 to Theorem II.5.1] that the Lorentz space $\Lambda_{\psi}\left(\mathbb{R}^{+}\right)$has order continuous norm and so the preceding remarks immediately imply that the Banach space dual of the noncommutative Lorentz space $\Lambda_{\psi}(\mathscr{M})$ is just the noncommutative Marcinkiewicz space $M_{\psi}(\mathscr{M})$, a result due to L. Ciach [Ci]. Similarly, if $M_{\psi}^{0}\left(\mathbb{R}^{+}\right)$denotes the linear subspace of $M_{\psi}\left(\mathbb{R}^{+}\right)$consisting of all $f \in M_{\psi}\left(\mathbb{R}^{+}\right)$for which

$$
\lim _{\alpha \rightarrow 0, \infty} \frac{1}{\psi(\alpha)} \int_{0}^{\alpha} \mu_{t}(f) d t=0,
$$


then it follows via [KPS, Theorem II.5.4] that $M_{\psi}^{0}(\mathscr{M})^{*}=\Lambda_{\psi}(\mathscr{M})$. In the case of trace ideals these results may be found in [GK, Chapter III.15].

The result which follows is (essentially) an extension of the LuxemburgLorentz theorem [Za1, Theorem 71.1] concerning the embedding of a Banach function space into its Köthe bidual. To smoothen the presentation, it is convenient to introduce the following terminology: if $\left\{x_{\alpha}\right\} \subseteq \widetilde{\mathscr{M}}$ then $\left\{x_{\alpha}\right\}$ is said to converge locally in measure to $x \in \widetilde{\mathscr{M}}$ if and only if $\left\{e x_{\alpha} e\right\}$ converges to exe for the measure topology on $\widetilde{\mathscr{M}}$ for all $e \in H(\mathscr{M})^{p}$.

Theorem 5.13. If $E \subseteq \widetilde{\mathscr{M}}$ is a properly symmetric Banach space, then the following statements are equivalent.

(i) The natural embedding of $E$ into $E^{\times \times}$is an isometry.

(ii) $0 \leq x_{\alpha} \uparrow_{\alpha} x \in E \Rightarrow\left\|x_{\alpha}\right\|_{E} \uparrow_{\alpha}\|x\|_{E}$.

(iii) If $\left\{x_{\alpha}\right\} \subseteq E$ converges locally in measure to $x \in E$ and $\left\|x_{\alpha}\right\|_{E} \leq 1$ for all $\alpha$ then $\|x\|_{E} \leq 1$.

Proof. (ii) $\Rightarrow$ (i). For $x \in H(\mathscr{M})$ and $n=1,2, \ldots$ define

$$
\|x\|_{E_{n}}=\inf \left\{\|y\|_{E}+n\|z\|_{1}: y, z \in H(\mathscr{M}), x=y+z\right\} .
$$

Each $\|\cdot\|_{E_{n}}, n=1,2, \ldots$, defines a norm on $H(\mathscr{M})$ and $\|\cdot\|_{E_{1}} \leq\|\cdot\|_{E_{2}} \leq$

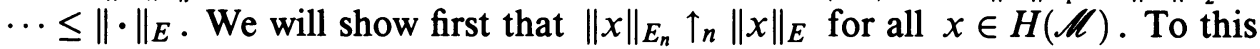
end, we observe that it is a simple consequence of Proposition 4.10 that

$$
\|x\|_{E_{n}}=\inf \left\{\|y\|_{E}+n\|z\|_{1}: y, z \in H(\mathscr{M}), x \prec \prec y+z\right\},
$$

for all $x \in H(\mathscr{M})$ and $n=1,2, \ldots$. From this it follows that $\left(H(\mathscr{M}),\|\cdot\|_{E_{n}}\right)$ is a symmetric rearrangement invariant operator space for each $n=1,2, \ldots$.

Let $e_{1}, \ldots, e_{N} \in \mathscr{M}^{p}$ be mutually orthogonal projections of finite trace and let

$$
\mathscr{N}=\left\{\sum_{j=1}^{N} \lambda_{j} e_{j}: \lambda_{1}, \ldots, \lambda_{N} \in \mathbb{C}\right\},
$$

which we may consider as a von Neumann algebra on the space $e H$, where $e=e_{1}+\cdots+e_{N}$. The restriction of $\tau$ to $\mathscr{N}$ is a finite trace on $\mathscr{N}$. It should be observed that if $x \in \mathscr{N}$ then its decreasing rearrangement as an element of $\mathscr{N}$ is the same as that when considered as an element of $\mathscr{M}$.

Now suppose that $x \in \mathscr{N}$. We claim that for all $n=1,2, \ldots$,

$$
\|x\|_{E_{n}}=\inf \left\{\|y\|_{E}+n\|z\|_{1}: y, z \in \mathscr{N}, x=y+z\right\} .
$$

Indeed, it is clear that the left-hand side is at most the infimum on the right. For the converse, suppose that $x=y+z$ with $y, z \in H(\mathscr{M})$. By Proposition 4.10, there exist $\hat{y}, \hat{z} \in \mathscr{N}$ such that $x=\hat{y}+\hat{z}$ and $\hat{y} \prec \prec y, \hat{z} \prec \prec z$, and so $\|\hat{y}\|_{E}+n\|\hat{z}\|_{1} \leq\|y\|_{E}+n\|z\|_{1}$, from which the claim follows. For $n=1,2, \ldots$, there exist $y_{n}, z_{n} \in \mathscr{N}$ such that $x=y_{n}+z_{n}$ and

$$
\left\|y_{n}\right\|_{E}+n\left\|z_{n}\right\|_{1} \leq\|x\|_{E_{n}}+\frac{1}{n} \leq\|x\|_{E}+\frac{1}{n} \text {. }
$$

Clearly $\left\|z_{n}\right\|_{1} \rightarrow 0$, and since $\operatorname{dim} \mathscr{N}<\infty$ this implies that $\left\|z_{n}\right\|_{E} \rightarrow 0$, so that $\left\|x-y_{n}\right\|_{E} \rightarrow 0$ as $n \rightarrow \infty$. Hence $\left\|y_{n}\right\|_{E} \rightarrow\|x\|_{E}$, and so $\|x\|_{E_{n}} \rightarrow\|x\|_{E}$ as $n \rightarrow \infty$. 
Now take any $0 \leq x \in H(\mathscr{M})$. There exists a sequence $\left\{x_{k}\right\}_{k=1}^{\infty}$ such that $0 \leq x_{k} \uparrow x$ in $H(\mathscr{M})$, where each $x_{k}$ is a finite linear combination of mutually orthogonal finite trace projections in $\mathscr{M}$. So, by the above observations, $\left\|x_{k}\right\|_{E_{n}} \uparrow_{n}\left\|x_{k}\right\|_{E}$ for all $k=1,2, \ldots$. Moreover, by the assumption on $\|\cdot\|_{E}$ we have $\left\|x_{k}\right\|_{E} \uparrow_{k}\|x\|_{E}$. Hence

$$
\|x\|_{E}=\sup _{k}\left\|x_{k}\right\|_{E}=\sup _{n, k}\left\|x_{k}\right\|_{E_{n}} \leq \sup _{n}\|x\|_{E_{n}} \leq\|x\|_{E}
$$

which shows that $\|x\|_{E_{n}} \uparrow_{n}\|x\|_{E}$.

Since $\|x\|_{E}=\||x|\|_{E}$ and $\|x\|_{E_{n}}=\||x|\|_{E_{n}}(n=1,2, \ldots)$ for all $x \in$ $H(\mathscr{M})$, we may conclude that $\|x\|_{E_{n}} \uparrow_{n}\|x\|_{E}$ for all $x \in H(\mathscr{M})$.

We denote by $H_{n}(\mathscr{M})$ the normed space $\left(H(\mathscr{M}),\|\cdot\|_{E_{n}}\right)$ for $n=1,2, \ldots$ If $\psi \in H_{n}(\mathscr{M})^{*}$, then $\psi \in H(\mathscr{M})^{*}$, and the inequality $\|\cdot\|_{E_{n}} \leq n\|\cdot\|_{1}$ implies that $\psi$ is normal. By Theorem 5.11, there exists $z_{\psi} \in G(\mathscr{M})$ such that

$$
\psi(x)=\tau\left(x z_{\psi}\right), \quad \text { for all } x \in H(\mathscr{M}) \text {. }
$$

The inequalities

$$
\left|\tau\left(x z_{\psi}\right)\right| \leq\|x\|_{E_{n}}\|\psi\| \leq\|x\|_{E}\|\psi\|, \quad \text { for all } x \in H(\mathscr{M}),
$$

now imply via Proposition 5.3 that $z_{\psi} \in E^{\times}$, and $\left\|z_{\psi}\right\|_{E^{\times}} \leq\|\psi\|$. Thus, if $x \in H(\mathscr{M})$, then

$$
\begin{aligned}
\|x\|_{E} & =\sup _{n}\|x\|_{E_{n}}=\sup _{n}\left\{|\psi(x)|: \psi \in H_{n}\left(M^{*}\right),\|\psi\| \leq 1\right\} \\
& \leq \sup \left\{|\tau(x y)|:\|y\|_{E^{\times}} \leq 1\right\}=\|x\|_{E^{\times \times}} .
\end{aligned}
$$

This suffices to show that the inequality $\|x\|_{E} \leq\|x\|_{E^{\times \times}}$continues to hold for all $x \in E$; in fact, if $x \in E$, then there exists a net $\left\{x_{\alpha}\right\} \subseteq H(\mathscr{M})$ with $0 \leq x_{\alpha} \uparrow_{\alpha}|x|$, and the assertion now follows from property (ii). Finally, we note that the reverse inequality $\|x\|_{E^{\times \times}} \leq\|x\|_{E}$ is trivial.

(i) $\Rightarrow$ (iii). Assume that $\left\{x_{\alpha}\right\} \subseteq E$, that $\left\{x_{\alpha}\right\}$ converges locally in measure to $x \in E$ and that $\left\|x_{\alpha}\right\|_{E} \leq 1$ for all $\alpha$. If $e \in H(\mathscr{M})^{p}$, there exists a sequence $\left\{x_{\alpha_{n}}\right\}$ such that at all points of continuity of $\mu($ exe $)$,

$$
\mu_{t}\left(e x_{\alpha_{n}} e\right) \rightarrow \mu_{t}(e x e)
$$

It follows from Fatou's Lemma that

$$
\int_{[0, \infty)} \mu_{t}(\text { exe }) \mu_{t}(y) d t \leq 1
$$

for each $e \in H(\mathscr{M})^{p}$ and $y \in H(\mathscr{M}),\|y\|_{E^{\times}} \leq 1$. The system $\{\mu($ exe $): e \in$ $\left.H(\mathscr{M})^{p}\right\}$ is upwards directed and it follows from Lemma 1.5 that $\mu(e x e) \uparrow_{e}$ $\mu(x)$ holds in $L^{0}\left(\mathbb{R}^{+}\right)$. Consequently

$$
\int_{[0, \infty)} \mu_{t}(x) \mu_{t}(y) d t \leq 1
$$

for each $y \in H(\mathscr{M}),\|y\|_{E^{\times}} \leq 1$ and (iii) now follows from the assertion of (i) that

$$
\|x\|_{E}=\sup \left\{\int_{[0, \infty)} \mu_{t}(x) \mu_{t}(y) d t: y \in H(\mathscr{M}),\|y\|_{E^{\times}} \leq 1\right\} .
$$

(iii) $\Rightarrow$ (ii). Suppose that $\left\{x_{\alpha}\right\} \subseteq E$ satisfies $0 \leq x_{\alpha} \uparrow_{\alpha} x \in E$. If $e \in$ $H(\mathscr{M})^{p}$ then it follows that $e\left(x-x_{\alpha}\right) e \downarrow_{\alpha} 0$ holds in $\widetilde{\mathscr{M}}_{0}$ and this implies by 
Lemma 3.5 that $\mu\left(e\left(x-x_{\alpha}\right) e\right) \rightarrow 0$. Consequently $\left\{x_{\alpha}\right\}$ converges locally in measure to $x$ and (iii) implies that $\|x\|_{E} \leq \sup _{\alpha}\left\|x_{\alpha}\right\|_{E}$. The reverse inequality is trivial and so (ii) follows.

Proposition 5.14. If $E \subseteq \widetilde{\mathscr{M}}$ is a properly symmetric Banach space, then the following statements are equivalent.

(i) The natural embedding of $E$ into $E^{\times \times}$is a surjective isometry.

(ii) $0 \leq x_{\alpha} \uparrow_{\alpha} \subseteq E$, $\sup _{\alpha}\left\|x_{\alpha}\right\|_{E}<\infty$ implies $x=\sup _{\alpha} x_{\alpha}$ exists in $E$ and $\|x\|_{E}=\sup _{\alpha}\left\|x_{\alpha}\right\|_{E}$.

(iii) If $\left\{x_{\alpha}\right\} \subseteq E$ converges locally in measure to $x \in \widetilde{\mathscr{M}}$ and if $\left\|x_{\alpha}\right\|_{E} \leq 1$ for all $\alpha$ then $x \in E$ and $\|x\|_{E} \leq 1$.

Proof. In view of Corollary 5.12 and Theorem 5.13 preceding, the equivalence (i) $\Leftrightarrow$ (ii) follows by noting that condition (ii) implies $E^{\times \times} \subseteq E$. This however follows immediately by observing that if $x \in E^{\times \times}$, then there exists $\left\{x_{\alpha}\right\} \subseteq H(\mathscr{M}) \subseteq E$ such that $0 \leq x_{\alpha} \uparrow_{\alpha}|x|$ holds in $\widetilde{\mathscr{M}}$, and from (ii) it follows that $x \in E$. The implications (iii) $\Rightarrow$ (ii), (i) $\Rightarrow$ (iii) follow from appropriate modifications of the proofs of the corresponding implications of Theorem 5.13.

The final result of this paper is a reflexivity criterion which in the commutative setting is a special case of a well-known theorem of $\mathrm{T}$. Ogasawara $[\mathrm{Za} 2$, Theorem 114.8].

Theorem 5.15. If $E \subseteq \widetilde{\mathscr{M}}$ is a properly symmetric Banach space, then the following statements are equivalent.

(i) $E$ is a reflexive Banach space.

(ii)(a) $0 \leq x_{\alpha} \uparrow_{\alpha} \subseteq E$, $\sup _{\alpha}\left\|x_{\alpha}\right\|_{E}<\infty$ implies $x=\sup _{\alpha} x_{\alpha}$ exists in $E$ and $\|x\|_{E}=\sup _{\alpha}\left\|x_{\alpha}\right\|_{E}$.

(b) The norms on $E$ and $E^{\times}$are order continuous.

Proof. (ii) $\Rightarrow$ (i). Order continuity of the norm on $E$ together with Theorem 5.11 implies that $E^{\times}$coincides with the Banach dual $E^{*}$, and order continuity of the norm on $E^{\times}$implies that the Banach dual $\left(E^{\times}\right)^{*}$ coincides with $E^{\times \times}$. Thus, it follows that $E^{* *}=E^{\times \times}$. Condition (ii)(a) together with Proposition 5.14 now implies that $E=E^{\times \times}$and it follows that $E$ is reflexive.

(i) $\Rightarrow$ (ii). Observe first that if $x_{\alpha} \downarrow_{\alpha} 0$ holds in $E$, then $\phi\left(x_{\alpha}\right) \downarrow 0$ holds for all $0 \leq \phi \in E^{*}$. In fact, since the positive cone of $E^{*}$ is generating, the net $\left\{x_{\alpha}\right\}$ is $\sigma\left(E, E^{*}\right)$-Cauchy, and hence $\sigma\left(E, E^{*}\right)$-convergent since the unit ball of $E$ is weakly compact. Dini's theorem now shows that convergence of the net $\left\{x_{\alpha}\right\}$ is uniform on the unit ball of $E^{*}$, and so the norm on $E$ is order continuous. The same argument now shows that the norm on $E^{\times}$is order continuous, and consequently $E=E^{* *}=E^{\times \times}$. Condition (ii)(a) is now an automatic consequence of Proposition 5.14.

Via Proposition 3.8 we now obtain the following consequence.

Corollary 5.16. Let $E\left(\mathbb{R}^{+}\right)$be a properly symmetric Banach function space on $\mathbb{R}^{+}$. If $E\left(\mathbb{R}^{+}\right)$is reflexive, then $E(\mathscr{M})$ is reflexive.

We remark that Proposition 5.15 and Corollary 5.16 preceding have been obtained independently by Goldstein and Sukochev [GS] in the special case that $\mathscr{M}$ is nonatomic and $\tau(1)<\infty$. 
We mention finally that topological properties such as weak sequential completeness and weak compactness in properly symmetric operator spaces can be characterized in terms of order properties. Such characterizations, well known in the theory of Banach lattices, may be found in [DDP4]. See also [GS].

\section{REFERENCES}

[AAP] C. Akemann, J. Anderson, and G. K. Pedersen, Triangle inequalities in operator algebras, Linear and Multilinear Algebra 11 (1982), 167-178.

[An] T. Ando, On fundamental properties of a Banach space with a cone, Pacific J. Math. 12 (1962), 1163-1169.

[BK] L. G. Brown and H. Kosaki, Jensen's inequality in semi-finite von Neumann algebras, J. Operator Theory 23 (1990), 3-19.

[Ca] A. P. Calderón, Spaces between $L^{1}$ and $L^{\infty}$ and the theorem of Marcinkiewicz, Studia Math. 26 (1966), 273-299.

[Ci] L. J. Ciach, On the conjugates of some operator spaces. I, Demonstratio Math. 18 (1985), 537-553.

[CS] V. I. Chilin and F. A. Sukochev, Symmetric spaces on semi-finite von Neumann algebras, Dokl. Akad. Nauk SSSR 13 (1990), no. 4, 811-815. (Russian)

[DDP1] P. G. Dodds, T. K. Dodds, and B. de Pagter, Non-commutative Banach function spaces, Math. Z. 201 (1989), 583-597.

[DDP2] _ A general Markus inequality, Proc. Centre Math. Anal. Austral. Nat. Univ. 24 (1989), 47-57.

[DDP3] _ Remarks on non-commutative interpolation, Proc. Centre Math. Anal. Austral. Nat. Univ. 24 (1989), 58-77.

[DDP4] _, Weakly compact subsets of symmetric operator spaces, Math. Proc. Cambridge Philos. Soc. 110 (1991), 169-182.

[Di] J. Dixmier, Von Neumann algebras, Math. Library, vol. 27, North-Holland, Amsterdam, 1981.

[DL] P. G. Dodds and C. J. Lennard, Normality in trace ideals, J. Operator Theory 16 (1986), 127-145.

[Fa1] T. Fack, Sur la notion de valeur caractéristique, J. Operator Theory 7 (1982), 307-333.

[Fa2] _ - Proof of the conjecture of A. Grothendieck on the Fuglede-Kadison determinant, J. Functional Anal. 50 (1983), 215-218.

[FK] T. Fack and H. Kosaki, Generalized s-numbers of $\tau$-measurable operators, Pacific J. Math. 123 (1986), 269-300.

[Fr] D. H. Fremlin, Stable subspaces of $L^{1}+L^{\infty}$, Proc. Cambridge Philos. Soc. 64 (1968), 625-643.

[Ga] D. J. H. Garling, On ideals of operators in Hilbert space, Proc. London Math. Soc. 17 (1967), 115-138.

[GK] I. C. Gohberg and M. G. Krein, Introduction to the theory of non-selfadjoint operators, Transl. Math. Monographs, vol. 18, Amer. Math. Soc., Providence, R.I., 1969.

[Gr] A. Grothendieck, Réarrangements de fonctions et inegalités de convexité dans les algèbres de von Neumann munies d'une trace, Seminaire Bourbaki, 1955, 113-01-113-13.

[GS] D. Sz. Goldstein and F. A. Sukochev, Conditions (A), (B), (C) and $\sigma\left(E, E^{\prime}\right)$-relative weak compactness of non-commutative symmetric spaces, VINITI, Tashkent, 1990, $26 \mathrm{pp}$. (Russian)

[Hi] F. Hiai, Majorization and stochastic maps in von Neumann algebras, J. Math. Anal. Appl. 127 (1987), 18-48.

[Ka] T. Kato, Perturbation theory for linear operators, Springer-Verlag, New York, Heidelberg, and Berlin, 1980. 
[KPS] S. G. Krein, Ju. I. Petunin, and E. M. Semenov, Interpolation of linear operators, Transl. Math. Monographs, vol. 54, Amer. Math. Soc., Providence, R.I., 1982.

[Le] M. Leinert, On integration with respect to a trace, J. Funct. Anal. (to appear).

[LS1] G. G. Lorentz and T. Shimogaki, Interpolation theorems for operators in function spaces, J. Funct. Anal. 2 (1968), 31-51.

[Lu] W. A. J. Luxemburg, Rearrangement invariant Banach function spaces, Queen's Papers in Pure and Appl. Math. 10 (1967), 83-144.

[Ne] E. Nelson, Notes on non-commutative integration, J. Funct. Anal. 15 (1974), 103-116.

[Ov1] V. I. Ovčinnikov, s-numbers of measurable operators, Funktsional. Anal. i Prilozhen. 4 (1970), 78-85. (Russian)

[Ov2] _ Symmetric spaces of measurable operators, Dokl. Akad. Nauk SSSR 191 (1970), 769-771; English transl., Soviet Math. Dokl. 11 (1970), 448-451.

[Ov3] _-, Symmetric spaces of measurable operators, Voronež. Gos. Univ. Trudy Naučn.-Issled. Inst. Mat. VGU 3 (1971), 88-107.

[PS] J. Peetre and G. Sparr, Interpolation and non-commutative integration, Ann. Mat. Pura Appl. 104 (1975), 187-207.

[Ru] G. I. Russu, On intermediate symmetrically normed ideals, Funktsional. Anal. i Prilozhen. 3 (1969), 94-95. (Russian)

[Ry] J. V. Ryff, Orbits of $L^{1}$-functions under doubly stochastic transformations, Trans. Amer. Math. Soc. 117 (1965), 92-100.

[Se] I. E. Segal, A non-commutative extension of abstract integration, Ann. of Math. (2) 57 (1953), 401-457.

[St] W. F. Stinespring, Integration theorems for gages and duality for unimodular groups, Trans. Amer. Math. Soc. 90 (1959), 15-56.

[Su] F. A. Sukochev, Symmetric spaces on finite von Neumann algebras, Thesis, Univ. of Tashkent, 1987.

[SZ] S. Strătilă and L. Zsidó, Lectures on von Neumann algebras, Abacus Press, Tunbridge Wells, 1979.

[Ta] M. Takesaki, Theory of operator algebras. I, Springer-Verlag, New York, Heidelberg, and Berlin, 1979.

[Te] M. Terp, $L^{p}$-spaces associated with von Neumann algebras, Notes, Copenhagen Univ., 1981.

[Ye1] F. J. Yeadon, Convergence of measurable operators, Math. Proc. Cambridge Philos. Soc. 74 (1973), 257-268.

[Ye2] — Non-commutative $L^{p}$-spaces, Math. Proc. Cambridge Philos. Soc. 77 (1975), 91-102.

[Ye3] _ Ergodic theorems for semifinite von Neumann algebras: II, Math. Proc. Cambridge Philos. Soc. 88 (1980), 135-147.

[Za1] A. C. Zaanen, Integration, North-Holland, Amsterdam, 1967.

[Za2] — Riesz spaces. II, North-Holland, Amsterdam, 1983.

(P. G. Dodds and T. K.-Y. Dodds) School of Information Science ANd Technology, The Flinders University of South Australia, GPO Box 2100, Adelaide 5001, Australia

E-mail address, P. G. Dodds: peter@ist.flinders.edu.au

E-mail address, T. K. Dodds: theresa@ist.flinders.edu.au

(Ben de Pagter) Department of Mathematics, Delft University of Technology, JulianalaAn 132, NL-2628 BL Delft, The Netherlands

E-mail address: depagter@dutiaw3.tudelft.nl 